\title{
ULTRASOUND-MODULATED \\ OPTICAL TOMOGRAPHY
}

\author{
A Dissertation \\ by \\ HAEWON NAM
}

\begin{abstract}
Submitted to the Office of Graduate Studies of Texas A\&M University in partial fulfillment of the requirements for the degree of

DOCTOR OF PHILOSOPHY
\end{abstract}

August 2002

Major Subject: Mathematics 


\title{
ULTRASOUND-MODULATED
}

\section{OPTICAL TOMOGRAPHY}

\author{
A Dissertation \\ by \\ HAEWON NAM \\ Submitted to Texas A\&M University \\ in partial fulfillment of the requirements \\ for the degree of \\ DOCTOR OF PHILOSOPHY
}

Approved as to style and content by:

\begin{tabular}{c}
\hline David C. Dobson \\
(Chair of Committee) \\
\hline $\begin{array}{c}\text { Jay R. Walton } \\
\text { (Member) }\end{array}$ \\
\hline $\begin{array}{c}\text { Lihong Wang } \\
\text { (Member) }\end{array}$
\end{tabular}

\begin{tabular}{c}
\hline Joseph E. Pasciak \\
(Member) \\
\hline William Rundell \\
(Head of Department)
\end{tabular}

August 2002

Major Subject: Mathematics 


\author{
ABSTRACT \\ Ultrasound-modulated \\ Optical Tomography. (August 2002) \\ Haewon Nam, B.S., Ewha Women's University; \\ M.S., Ewha Women's University \\ Chair of Advisory Committee: Dr. David C. Dobson
}

Ultrasound-modulated optical tomography is modeled by a linear integral equation and an inverse problem involving a diffusion equation in $n$ spatial dimensions, $n=2$, 3. Based on measured data, the optical absorption coefficient $\mu$ is reconstructed inside of a given domain. We make a two-step mathematical model. First, we solve a linear integral equation. Assuming the energy fluence rate has been recovered from the previous equation, the absorption coefficient $\mu$ is then reconstructed by solving an inverse problem. Numerical experiments are presented for the case $n=2$. Two methods are used for the numerical experiments, gradient descent and levelset. At the end, advantages and disadvantages of those two methods are mentioned. 
To my parents and my husband, Hyun Ho Lee 


\section{ACKNOWLEDGMENTS}

The years I spent learning knowledge, conducting the research, and writing this dissertation were filled with many intellectual and emotional challenges. I would like to thank and mention those whose support has contributed to the strengths of my research.

I would like to thank Dr. David C. Dobson, chair of my committee, for providing me the opportunity to be involved in this wonderful field of the research. I would like to thank Dr. Lihong Wang for his advice and for providing me with an understanding of the fundamentals of physics and biological tomography. I would also like to thank Dr. Jay Walton and Dr. Joe Pasciak for many helpful discussions and reviews regarding my research results.

I must thank my family and friends for their love and support throughout my graduate education:my parents, parents-in-law, brothers, brother-in-law, sisters-inlaw, and my husband. Finally, I thank God for everything. 


\section{TABLE OF CONTENTS}

CHAPTER

INTRODUCTION . . . . . . . . . . . . . . . . . . 1

II

BACKGROUND INFORMATION . . . . . . . . . . . 7

A. Notation and Concepts ............. 7

B. Boltzmann Equation .............. 10

C. Diffusion Approximation . . . . . . . . . . . . 11

D. Interaction of Optical Field and Ultrasound Column . . . . 13

III MATHEMATICAL MODEL . . . . . . . . . . . . . 16

A. Recovering the Optical Field . . . . . . . . . . . 16

B. Recovering the Absorption Coefficient . . . . . . . . 17

C. Minimization Problem . . . . . . . . . . . 18

1. Properties of the Forward Map . . . . . . . . . . 19

2. Compactness of Admissible Solution Space . . . . . 21

3. Existence and Uniqueness of Solutions . . . . . . . 22

IV NUMERICAL RESULTS . . . . . . . . . . . . . . 32

A. Gradient Descent Method . . . . . . . . . . . 32

1. Gradient Descent . . . . . . . . . . . . 32

2. Minimal Total Variation Regularization . . . . . 36

B. Levelset Method . . . . . . . . . . . . . . . . . 39

1. Levelset . . . . . . . . . . . . . . 40 40

2. Levelset Method with Total Variation Regularization . 46

V SUMMARY AND CONCLUSIONS ............ 51

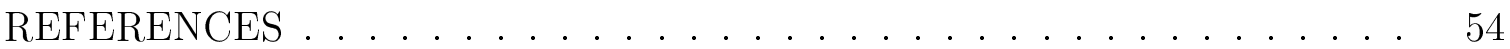

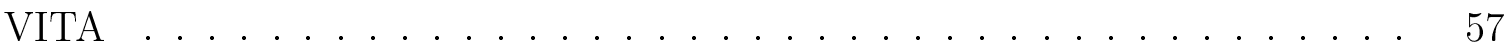




\section{LIST OF FIGURES}

FIGURE

Sketch of UMOT . . . . . . . . . . . . . . . . . . 3

2 Sketch of the Experimental Setup. . . . . . . . . . . . . . 4

3 Sketch of the Boundary .................. 18

$4 \quad$ Result of the Gradient Descent Method . . . . . . . . . . . . . 35

$5 \quad$ Blocky Model . . . . . . . . . . . . . . . . 38

$6 \quad$ Volume Fraction . . . . . . . . . . . . . . . . . . . . 42

$7 \quad$ Reconstruction of A-shaped Block. . . . . . . . . . . . . . 43

8 Initial Levelset Function . . . . . . . . . . . . . . . . . . . 44

$9 \quad$ Final Levelset Function _. . . . . . . . . . . . . . . . . 45

10 Blocky Solution and Blurred Noise . . . . . . . . . . . 47

11 Levelset Reconstructions with Noisy Data . . . . . . . . . . . . . 48

12 Simple Block Reconstructions with Total Variation Regularization . . 49

13 Complicated Shape Reconstruction with Total Variation

Regularization ................. 50 


\section{CHAPTER I}

\section{INTRODUCTION}

In this thesis we study ultrasound-modulated optical tomography (UMOT), a relatively new imaging technology which attempts to combine some of the advantages of ultrasound imaging with those of optical tomography. We will make a mathematical model of UMOT and study mathematical properties of the model, along with reconstruction algorithms. Our ultimate goal is to make a precise reconstruction of physically important material parameters using UMOT.

Optical imaging of biological tissues has been an active research area recently. Since biological tissues are highly scattering media, sophisticated reconstruction methods are required. The main advantages of optical tomography are that it has a good contrast between healthy and diseased tissue and it is inexpensive, nonionizing, and noninvasive. The primary disadvantage is that reconstructions are generally unstable and have low resolution. Ultrasound is essentially harmless, noninvasive, nonionizing, inexpensive, and has good resolution. Unfortunately, ultrasound has low contrast between healthy and diseased tissue, thus it is of limited usefulness for diagnosing conditions like breast cancer. Ultrasound-modulated optical tomography attempts to combine the good resolution of ultrasound with the high contrast of optical imaging. In ultrasound-modulated optical tomography, some of the diffuse light inside the biological tissue is modulated by a partially-localized ultrasonic wave. Modulated photons carry the ultrasonic frequency. The intensity of tagged photons for a given position of the ultrasonic column can be used to derive the optical absorption coefficient at that point [28]. As illustrated in Figure 1, we denote $E$ the aperture of

The journal model is SIAM Journal on Applied Mathematics. 
CCD camera which is used for measuring modulated signals. Ultrasound-modulated tomography was developed by Lihong Wang [25, 27, 28]. The experimental setup is as shown in Figure 2. The $\mathrm{Z}$ axis lies along the optical axis pointing to the CCD camera. The $\mathrm{Y}$ axis lies along the acoustic axis pointing towards the sample. The $\mathrm{X}$ axis is perpendicular to both the acoustic and the optical axes. The ultrasonic column is generated by a focused ultrasonic transducer. The tissue sample is partially immersed in water for good acoustic coupling. Two function generators are used to excite the ultrasonic transducer and to modulate the diode laser. The two function generators and the CCD camera are controlled by a personal computer [28].

In Chapter 2, we describe background information regarding models of photon transport, and the interaction between acoustic and optical fields. This information is used to derive a mathematical model of this inverse problem. This problem can be divided into two steps. The first step comes from the effect of the ultrasound transducer $[14,17]$. The second step comes from diffusion theory. The diffusion equation is originated from the Boltzmann equation, which describes photon transport in the medium $[19,26]$. The first section of this chapter gives some notation and basic definitions and theorems. The second part of this chapter explains the Boltzmann equation, which is also called the linear transport equation. Instead of using the Boltzmann equation directly, we simplify using the diffusion equation in this thesis. The third part of this chapter shows the derivation of diffusion equation from the Boltzmann equation. We also study the interaction between acoustic and optical fields, explaining the first step of our model.

In Chapter 3, we study some mathematical properties of the model. We set a minimization problem and study the properties of the objective function. We show the existence and local uniqueness of solutions to this problem. To formulate the diffusion equation, we divide the boundary into two parts. One part is where the 

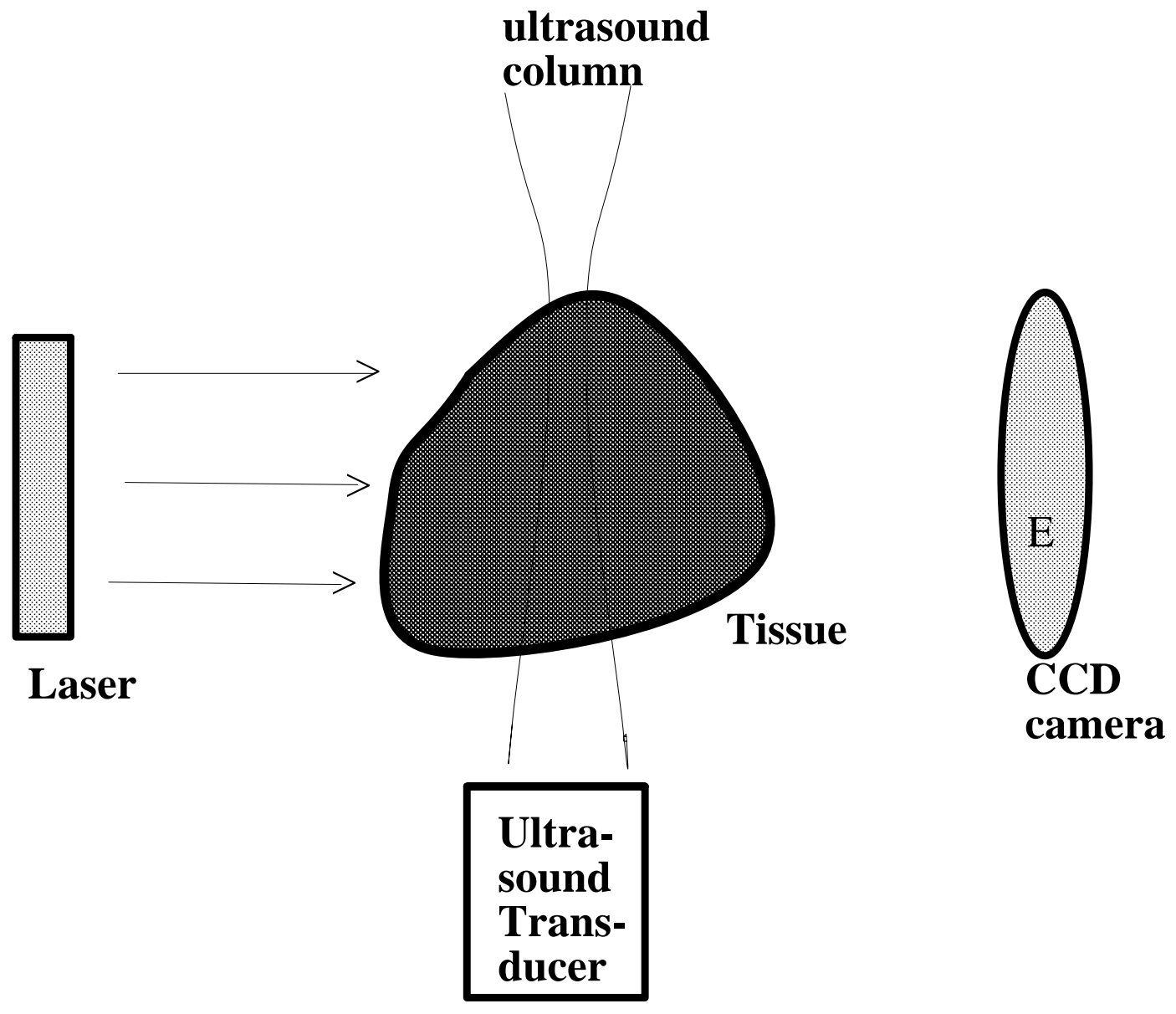

Fig. 1. Sketch of UMOT 


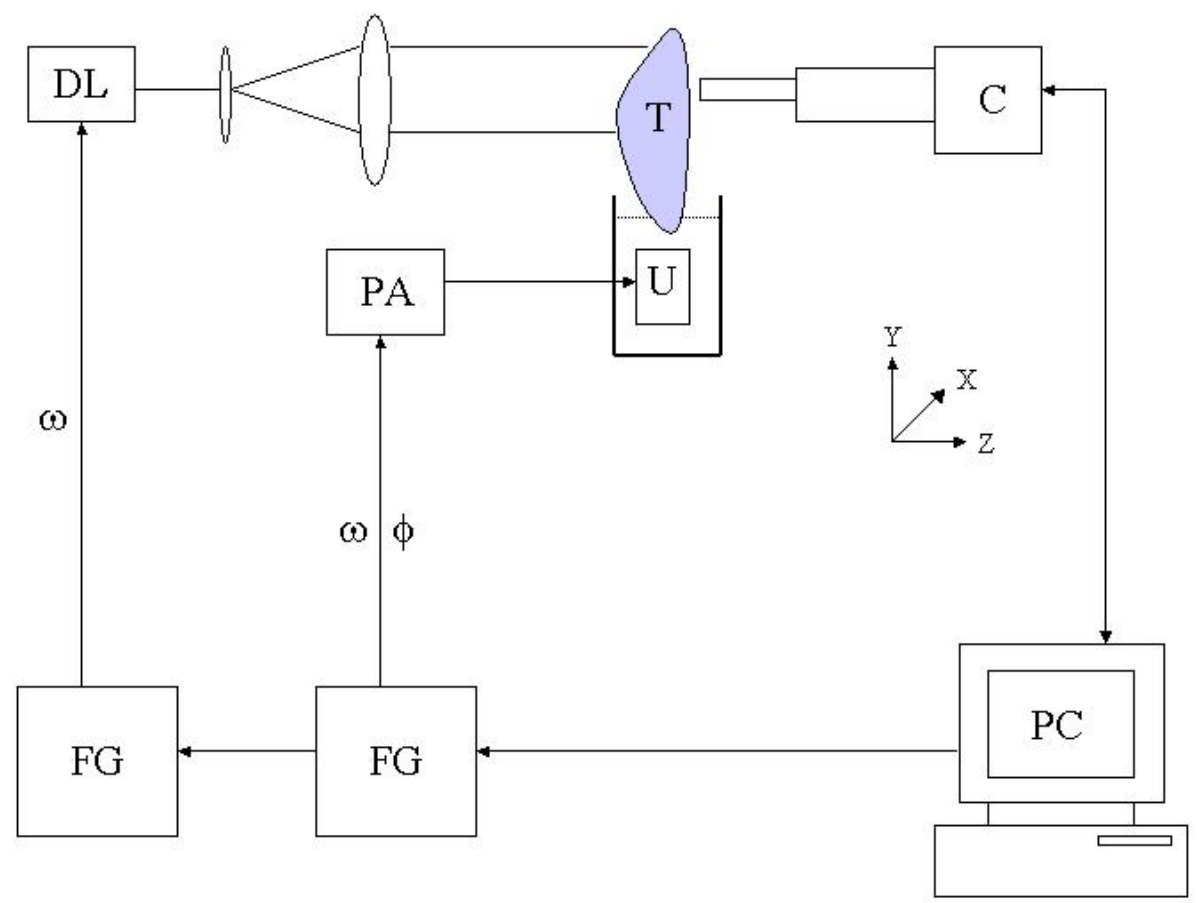

Fig. 2. Sketch of the Experimental Setup.

DL, diode laser; C, CCD camera; U, Ultrasonic Transducer; FG's, Function Generator; PA, Power Amplifier; T, Tissue Sample; PC, Personal Computer; $\phi$, Initial Phase of the Speckle 
optical field comes in; the other incorporates a transmission condition. We show the continuity, and differentiability of the "forward map" which takes a given absorption coefficient to a solution of the diffusion problem. We obtain the existence of solutions to the minimization problem by showing that the space of admissible absorption coefficients is compact. The uniqueness problem is not as easy as the existence. In [21] it is proved that $\phi$ uniquely determines $\alpha$, if $\nabla \cdot \alpha \nabla \phi=0$ and some properties of $\phi$ and $\alpha$ are satisfied. Knowles improved uniqueness for more general case [15]. But our model is more complicated than this. We use the analyticity of the objective function to show local uniqueness. We show that the objective is analytic on the small neighborhood. Then we show local uniqueness using the Taylor series.

In Chapter 4, we present numerical results of certain reconstruction methods applied to UMOT. We use two approaches to attempt to obtain better reconstructions. The first section of this chapter shows reconstruction results from the gradient descent method. The gradient descent method is one of the most famous function minimization methods. In gradient descent, the derivative of the function to be minimized is used to calculate an adjoint object called the "gradient". Once we calculate the derivative of the function, then the approximate solution is moved in the negative direction of the gradient. That will reduce the value of the function. We combine the gradient descent method with a total variation regularization in an attempt to recover blocky shapes, following methods described in $[4,5,6]$. In the second section we use the levelset method to reconstruct the absorption coefficient. We assume that the absorption coefficient is a characteristic function rather than a general $L^{\infty}$ function to use the levelset method. The levelset method was first given by Osher and Sethian for describing the motion of curves and surfaces [20]. Dorn [9] used the levelset method for optical tomography. He used the Boltzmann equation directly rather than the diffusion approximation, but did not consider the case of ultrasound 
modulation. The levelset method is fast and accurate compared to gradient descent. It typically converges to the actual solution within 30 iterations. But it is unstable in the sense that if we add a small amount of noise in the data, then the reconstruction deteriorates. We again use total variation as a regularization to improve stability.

The final Chapter summarizes the results of this paper and indicates some directions for future research. 


\section{CHAPTER II}

\section{BACKGROUND INFORMATION}

This chapter provides background information on mathematical models for photon transport in biological media. This information will be used later in the thesis to model the ultrasound-modulated optical tomography problem. This chapter also introduces notations and mathematical concepts to be used later.

\section{A. Notation and Concepts}

Throughout this thesis $\Omega$ will denote an open, bounded domain in $\mathbb{R}^{n}, n=2,3$ with Lipschitz continuous boundary.

Definition A.1 Let $k$ be a nonnegative integer or $k=\infty . C^{k}(\Omega)$ is the set of functions having all derivatives of order less than or equal to $k$ continuous on $\Omega$ [11].

Definition A.2 Let $G$ be an open set in $\mathbb{R}^{n}, n \geq 1$. We let $\mathcal{L}^{2}(G)$ denote the classical Banach space consisting of measurable functions on $G$ that are square integrable.

$$
\mathcal{L}^{2}(G)=\left\{u:\left.\Omega \rightarrow \mathbb{R}\left|\int_{G}\right| u\right|^{2} d x<\infty\right\} .
$$

Furthermore, for all $u, v \in \mathcal{L}^{2}(G)$ we set the inner product of $\mathcal{L}^{2}$ is defined by

$$
<u, v>_{L^{2}(G)}:=\int_{G} u v d x,
$$

and the norm $\|u\|_{\mathcal{L}^{2}}^{2}:=<u, u>_{L^{2}(G)}[11]$.

Definition A.3 Let $G$ be a nonempty open set in $\mathbb{R}^{n}, n \geq 1$. The Sobolev space $\mathcal{H}^{1}(G)$ consists precisely of all functions

$$
u \in \mathcal{L}^{2}(G)
$$


that have generalized derivatives

$$
\partial_{j} u \in \mathcal{L}^{2}(G) \text { for all } j=1, \ldots n \text {. }
$$

Furthermore, for all $u, v \in \mathcal{H}^{1}(G)$, we set the inner product

$$
<u, v>_{1}:=\int_{G}\left(u v+\sum_{j=1}^{n} \partial_{j} u \partial_{j} v\right) d x,
$$

and the norm $\|u\|_{\mathcal{H}^{1}}^{2}:=<u, u>_{1}[11]$.

More generally, we can define as follows.

Definition A.4 Let us denote by $W^{k}(\Omega)$ the set of $k$ times weakly differentiable functions on $\Omega$. The $W^{k, p}(\Omega)$ spaces are Banach spaces defined as

$$
W^{k, p}(\Omega)=\left\{u \in W^{k}(\Omega) ; D^{\alpha} u \in \mathcal{L}^{p}(\Omega) \text { for all }|\alpha| \leq k\right\}
$$

with norm $\|u\|_{W^{k, p}(\Omega)}=\left(\int_{\Omega} \sum_{|\alpha| \leq k}\left|D^{\alpha} u\right|^{p} d x\right)^{1 / p}[11]$.

Definition A.5 (Fréchet Derivative[22]) Let $X$ and $Y$ be Banach spaces and $x_{0}$ be a point in $X$. Let $F$ be a mapping from a neighborhood of $x_{0}$ into $Y$. Then $F$ is called (Fréchet) differentiable at $x_{0}$ if there exists a linear operator $A$ from $X$ to $Y$ with the property that

$$
F(x)=F\left(x_{0}\right)+A\left(x-x_{0}\right)+G(x),
$$

where

$$
\lim _{x \rightarrow x_{0}}\|G(x)\|_{Y} /\left\|x-x_{0}\right\|_{X}=0
$$

If such an $A$ exists, we call it the Fréchet derivative of $F$ at $x_{0}$. We call a function $F$ differentiable if it is differentiable at all points of its domain. 
We shall use familiar notations from calculus, such as $F^{\prime}\left(x_{0}\right)$ or $D F\left(x_{0}\right)$ to denote Fréchet derivatives.

Definition A.6 (Normal Operator[16]) A bounded linear operator $T: H \rightarrow H$ on a Hilbert space $H$ is said to be normal if $T T^{*}=T^{*} T$.

Definition A.7 (Compact Operator[29]) Let $X$ and $Y$ be normed spaces over $\mathbb{R}$ or $\mathbb{C}$. The operator

$$
A: M \subseteq X \rightarrow Y
$$

is called compact if and only if

1. A is continuous, and

2. A transforms bounded sets into relatively compact sets.

Throughout this thesis we define $X$ as a subset of $\mathcal{L}^{\infty}(\Omega)$ such that

$$
\begin{aligned}
X= & \left\{\mu \in \mathcal{L}^{\infty}(\Omega): 0 \lesseqgtr \mu_{1} \leq \mu(x) \leq \mu_{2} \lesseqgtr \infty, \mu\right. \text { is Lipschitz continuous with } \\
& \operatorname{Lip}(\mu) \leq c \text { and } \mu=q \text { on } \partial \Omega\}
\end{aligned}
$$

for some given Lipschitz continuous function $q$, and a given constant $c$.

In this thesis we use the gradient descent method for the numerical results. The gradient descent method is a function optimization method which uses the derivative of a function and the idea of steepest descent. According to Definition A.5, the derivative of a function $J: X \rightarrow \mathbb{R}$ at the point $x_{0} \in X$ is a linear operator $A$ mapping $X$ into $\mathbb{R}$. If $X$ is a Hilbert space, then by the Riesz representation, the derivative operator must have a "representative" $a \in X$, i.e. $A x=<a, x>_{X}$ for all $x \in X$. The representative $a$ is usually called the gradient. We will denote $D J\left(x_{0}\right)=a$. The derivative of a function in a particular unit direction is the slope. The slope is maximized in the gradient direction, and minimized in the negative 
gradient direction. Moving slightly in the direction of the negative gradient reduces the value of the function [3]. The gradient descent method is described as follows;

1. Let $J$ be the function to be minimized. Let $x_{0}$ be the initial guess. Compute the gradient of $J, D J\left(x_{0}\right)$. Set $\mathrm{n}=0$.

2. update $x_{n+1}=x_{n}-h D J\left(x_{n}\right)$, where $\mathrm{h}$ is a step size which must be chosen so that we don't take too big or too small of a step. Too big step will overshoot the function minimum and too small step will result in slow convergence.

3. Update $n=n+1$. Repeat the above two steps until we converge to a local minimum of the function $J(x)$.

The gradient descent method is conceptually straightforward. With proper stepsize control $h$, and mild assumptions on $J$, it can be shown to converge to a local minimum $[3]$.

\section{B. Boltzmann Equation}

The Boltzmann transport equation describes the migration of photons in biological tissues: $[7,8,9,12]$

$$
S(r, \zeta)-\zeta \nabla \Psi(r, \zeta)-\left(\mu_{a}+\mu_{s}\right) \Psi(r, \zeta)+\mu_{s} \int_{4 \pi} \Psi\left(r, \zeta^{\prime}\right) p\left(\zeta \cdot \zeta^{\prime}\right) d \zeta^{\prime}=0
$$

where $r \in \mathbb{R}^{3}$ is the position vector and $\zeta \in \mathbb{R}^{3}$ a unit vector pointing in the direction of photon propagation. Here, $\Psi(r, \zeta)$ is the energy radiance in units of $W \mathrm{~cm}^{-2} s r^{-1}$. The source term $S(r, \zeta)$ is the power injected into a solid angle centered on $\zeta$ in a unit volume at $r$. The quantities $\mu_{a}$ and $\mu_{s}$ are the absorption and scattering coefficients which are the inverse of the mean free path for absorption and scattering, respectively. The phase function $p\left(\zeta, \zeta^{\prime}\right)$ is the probability that during a scattering event a photon 
with direction $\zeta^{\prime}$ is scattered in the direction $\zeta$ per unit receiving solid angle. The phase function usually depends only on the change in direction of photon. Therefore $p\left(\zeta, \zeta^{\prime}\right)=p\left(\zeta \cdot \zeta^{\prime}\right)$ and the anisotropy, the average of the cosine of the scattering angle $g=\int_{4 \pi}\left(\zeta \cdot \zeta^{\prime}\right) p\left(\zeta \cdot \zeta^{\prime}\right) d \zeta$. Let $\varphi(r)=\int_{4 \pi} \Psi(r, \zeta) d \zeta$. The function $\varphi$ is the energy fluence rate with units of $W / \mathrm{cm}^{2}$. The quantity $J(r)=\int_{4 \pi} \zeta \Psi(r, \zeta) d \zeta$ is the flux or current density. It is a net energy flow per unit area per unit time. The transport equation model can be used to perform accurate computer simulations of photon transport in biological tissue [25].

\section{Diffusion Approximation}

We use the diffusion approximation to simplify subsequent theory $[7,8,9,19]$. In the diffusion approximation, it is assumed that photon scattering is the dominant process in the material, which is mathematically expressed as $\mu_{a} / \mu_{s} \ll 1$.

Our derivation here follows [26]. In the diffusion approximation, the radiance is expanded in spherical harmonics truncated at the first order. Thus the radiance can be rewritten as $[26]$

$$
\Psi(r, s)=\sum_{n=0}^{1} \sum_{m=-n}^{n} L_{n, m}(r) Y_{n, m}(s),
$$

where $r \in \mathbb{R}^{3}$ is the position vector and $s \in \mathbb{R}^{3}$ a unit vector pointing in the direction of photon propagation. $Y_{n, m}(s)$ denotes spherical harmonic function as

$$
\begin{aligned}
Y_{n, m}(s) & =Y_{n, m}(\theta, \phi) \\
& =(-1)^{m} \sqrt{\frac{(2 n+1)(n-m) !}{4 \pi(n+m) !}} P_{n, m}(\cos \theta) \exp (i m \phi),
\end{aligned}
$$

where $P_{m, n}(\cos \theta)$ denotes the associated Legendre polynomial. Here $\theta$ and $\phi$ are the radial and azimuthal angles associated with $s$, respectively. By substituting the 
expansion of $\Psi$ into $\varphi(r)=\int_{4 \pi} \Psi(r, s) d s$, we obtain

$$
\varphi(r)=4 \pi L_{0,0}(r) Y_{0,0}(s)
$$

Let $J(r)$ be the flux. By substituting the expansion of $\Psi$ into $J(r)=\int_{4 \pi} s \Psi(r, s) d s$, we get

$$
J(r) \cdot \zeta=\frac{4 \pi}{3} \sum_{m=-1}^{1} L_{1, m}(r) Y_{1, m}(\zeta)
$$

Therefore

$$
\Psi(r, \zeta)=\frac{1}{4 \pi} \varphi(r)+\frac{3}{4 \pi} J(r) \cdot \zeta
$$

The phase function depends only on the change in direction of photon and can be expanded in Legendre polynomials as

$$
\begin{aligned}
p\left(\zeta \cdot \zeta^{\prime}\right) & =\sum_{n=0}^{1} \frac{2 n+1}{4 \pi} g_{n} P_{n}\left(\zeta \cdot \zeta^{\prime}\right) \\
& =\sum_{n=0}^{1} \sum_{m=-n}^{n} g_{n} \bar{Y}_{n, m}\left(\zeta^{\prime}\right) Y_{n, m}(\zeta)
\end{aligned}
$$

where $P_{n}=P_{n, 0}$ are Legendre polynomials and $\bar{Y}_{n, m}$ denotes the complex conjugate of $Y_{n, m}$. By using $\int_{4 \pi} p\left(\zeta \cdot \zeta^{\prime}\right)=1$ and $\int_{4 \pi} \zeta \cdot \zeta^{\prime} p\left(\zeta \cdot \zeta^{\prime}\right)=1$, we obtain $g_{0}=1$ and $g_{1}=g$, respectively. Thus we have

$$
p\left(\zeta \cdot \zeta^{\prime}\right)=\frac{1}{4 \pi}(1+3 g \cos \theta)
$$

By substituting (2.2) and (2.4) into the Boltzmann equation (2.1) and integrating over the full $4 \pi$ solid angle, we obtain

$$
\mu_{a} \varphi(r)+\nabla \cdot J(r)=S(r)
$$

Similarly, using (2.2) and (2.4) in the Boltzmann equation (2.1), multiplying by $s$ and 
integrating over the solid angle, we have

$$
\left(\mu_{a}+\mu_{s}^{\prime}\right) J(r)+\frac{1}{3} \nabla \varphi(r)=0
$$

where $\mu_{s}^{\prime}=(1-g) \mu_{s}$ which is called the reduced scattering coefficient. Thus we obtain

$$
J(r)=-\frac{1}{3\left(\mu_{a}+\mu_{s}^{\prime}\right)} \nabla \varphi(r),
$$

which is called Fick's law. Therefore we have

$$
-\nabla \cdot \frac{1}{3\left(\mu_{a}+\mu_{s}^{\prime}\right)} \nabla \varphi(r)+\mu_{a} \varphi(r)=S(r)
$$

For simplicity, let us denote $\mu_{a}=\mu$ and $\mu_{s}^{\prime}=\mu_{s}$.

The diffusion equation (2.6) is very practical when used in the inverse transport problem arising in optical tomography. It is well understood and can be discretized efficiently. On the other hand, the transport model (2.1) is more general and can be applied in situations where the diffusion approximation fails to give good results [7].

\section{Interaction of Optical Field and Ultrasound Column}

We are interested in the interaction of an acoustic beam with an optical field inside a body [17]. The correlation function is given by [14];

$$
G(r, \tau) \approx \frac{-k^{2}}{3 l^{3}} \int I\left(r^{\prime}\right) P\left(r, r^{\prime}\right) A^{2}\left(r^{\prime}\right)\left(1-\cos \omega_{s} \tau\right) \ll \exp \left(i \triangle \phi^{\prime}(\tau)\right) \gg d r^{\prime}
$$

where $r \in \mathbb{R}^{3}$ is the position vector, $\mathrm{k}$ is the optical wave vector, $\omega_{s}$ is the frequency, $A(r)$ is the amplitude of the displacement. The quantity $A(r)$ is the distance the material at point $r$ gets displaced by the acoustic field. It is related to the acoustic pressure. Here $I\left(r^{\prime}\right)$ is the intensity of the optical field. For our diffusion model, $I\left(r^{\prime}\right)=\varphi\left(r^{\prime}\right)$ where $\varphi$ satisfies (2.6). The quantity $l$ is an average distance of the 
transport mean free path, $\tau$ is a time. The function $P\left(r, r^{\prime}\right)$ is the probability of arriving at $r$ from $r^{\prime}$. The function

$$
\ll \exp \left(i \triangle \phi^{\prime}(\tau)\right) \gg=\prod_{j=1}^{n(\alpha)}<\exp \left(i q_{j} \cdot \Delta r_{j}^{\prime}\right)>
$$

where $\triangle r_{j}^{\prime}$ is the fluctuation in the displacement of the $j$ th scatterer related to its random motion, and $q_{j}$ is the scattering vector. Here $\alpha$ is the path between the observation times $t$ and $t+\tau$ with the displacement of $n$ scatterers. The angle brackets indicate the average over time. The term $\ll \exp \left(i \triangle \phi^{\prime}(\tau)\right) \gg$ would be observed in the absence of the acoustic field, where $\phi$ is the part of the accumulated phase that is associated with the path length.

By taking the average over time $\tau$, we can rewrite (2.7) as

$$
G_{1}(r)=c \int P\left(r, r^{\prime}\right) A^{2}\left(r^{\prime}\right) \varphi\left(r^{\prime}\right) d r^{\prime}
$$

where the $c$ is a constant. Here, the quantity $G_{1}(r)$ is the time-averaged correlation at $r$. Let $E$ be the aperture of the detector used for measuring the modulated signal. Then the measurement taken with the acoustic column located at $x=0$ is

$$
\begin{aligned}
\int_{E} G_{1}(r) d r & =c \int_{E} \int_{\Omega} P\left(r, r^{\prime}\right) A^{2}\left(r^{\prime}\right) \varphi\left(r^{\prime}\right) d r^{\prime} d r \\
& =c \int_{\Omega} P\left(r^{\prime}\right) A^{2}\left(r^{\prime}\right) \varphi\left(r^{\prime}\right) d r^{\prime}
\end{aligned}
$$

where $P\left(r^{\prime}\right)=\int_{E} P\left(r, r^{\prime}\right) d r$. Assuming that the acoustic column does not change shape upon translation, the measurement at arbitrary location $x$ is

$$
G(x)=c \int_{\Omega} P\left(r^{\prime}\right) A^{2}\left(r^{\prime}-x\right) \varphi\left(r^{\prime}\right) d r^{\prime}
$$

A quantity related to the field correlation function can be measured by beating the transmitted signal with a local oscillator field derived from the laser [14]. For our 
purpose, we assume that the quantity $G(x)$ itself is measured at each acoustic column position $x$. 


\section{CHAPTER III}

\section{MATHEMATICAL MODEL}

Given some domain $\Omega \subset \mathbb{R}^{n}, \mathrm{n}=2$ or 3 , with Lipschitz boundary, we are interested in the problem of recovering the absorption coefficient $\mu$ inside $\Omega$ from the measured ultrasound-modulated photons captured outside $\Omega$. In the following we assume that $\mathrm{n}=2$. This problem can be divided into two steps, discussed next.

\section{A. Recovering the Optical Field}

The first step is to recover the optical field $\varphi$ from the measured data $G(x)$ defined in (2.9). Let us define the convolution operator

$$
(T u)(x)=\int_{\Omega} g(x-y) u(y) d y
$$

where $u(y)=P(y) \varphi(y)$ and $g(z)=c A^{2}(-z)$, where $A$ and the constant $c$ are from $(2.9)$.

Recall that the quantity $(T u)(x)$ is from the interaction of a narrow acoustic beam with an optical field inside the medium [14]. We assume that the acoustic column can be translated anywhere within the medium, so that $x \in \Omega$. The problem is to recover the optical field $\varphi$ from $(T u)(x)$. Observe that (3.1) is a convolution of $g$ and $u$. Let $g_{0}$ be a given measurement. Then (3.1) becomes

$$
(T u)(x)=(g * u)(x)=g_{0}(x) .
$$

The displacement $A(x)$ is the solution to a simple acoustic wave equation, hence the kernel $g$ is a continuous function with $g \in \mathcal{L}^{2}(\Omega)$. Then the function $T$ is a linear bounded compact operator from $\mathcal{L}^{2}(\Omega)$ into $\mathcal{L}^{2}(\Omega)[29]$. Also, there exists the adjoint operator $T^{*}$ [29]. Instead of solving (3.2) directly, we consider a regularized form of 
the normal equation [10]

$$
\left(T^{*} T+\epsilon I\right) u=T^{*} g_{0}
$$

where $\epsilon$ is a small positive number. Then the existence and uniqueness of solutions for (3.3) follows immediately from the fact that $T^{*} T+\epsilon I$ is a normal operator [16]. As a self-adjoint linear equation, problem (3.3) can also be discretized and solved for example by the conjugate gradient method. For the remainder of this work, we will assume that (3.3) has been solved to yield an approximate optical field

$$
\tilde{\varphi}=\left(T^{*} T+\epsilon I\right)^{-1} T^{*} g_{0} / P
$$

B. Recovering the Absorption Coefficient

The second step is to recover the absorption coefficient from the function $\tilde{\varphi}$ from the first step. The class of "admissible" absorption coefficients $X$ should be chosen to include all possible expected $\mu$.

Consider the model

$$
-\nabla \cdot \frac{1}{3\left(\mu+\mu_{s}\right)} \nabla \varphi+\mu \varphi=S
$$

The boundary of $\Omega$ can be written as a disjoint union, $\partial \Omega=\Gamma_{0} \cup \Gamma_{1}$. As illustrated in Figure 3, $\Gamma_{0}$ is the part of the boundary which the laser beam illuminates and $\Gamma_{1}$ denotes the other part. Note that the source term $S=0$ in $\Omega$. Define $\gamma(\mu)=\frac{1}{3\left(\mu+\mu_{s}\right)}$. Then, the diffusion equation becomes

$$
\left\{\begin{array}{l}
-\nabla \cdot \gamma(\mu) \nabla \varphi+\mu \varphi=0 \text { in } \Omega, \\
\gamma(\mu) \frac{\partial \varphi}{\partial n}=f \text { on } \Gamma_{0}, \\
\gamma(\mu) \frac{\partial \varphi}{\partial n}+\alpha \varphi=0 \text { on } \Gamma_{1} .
\end{array}\right.
$$




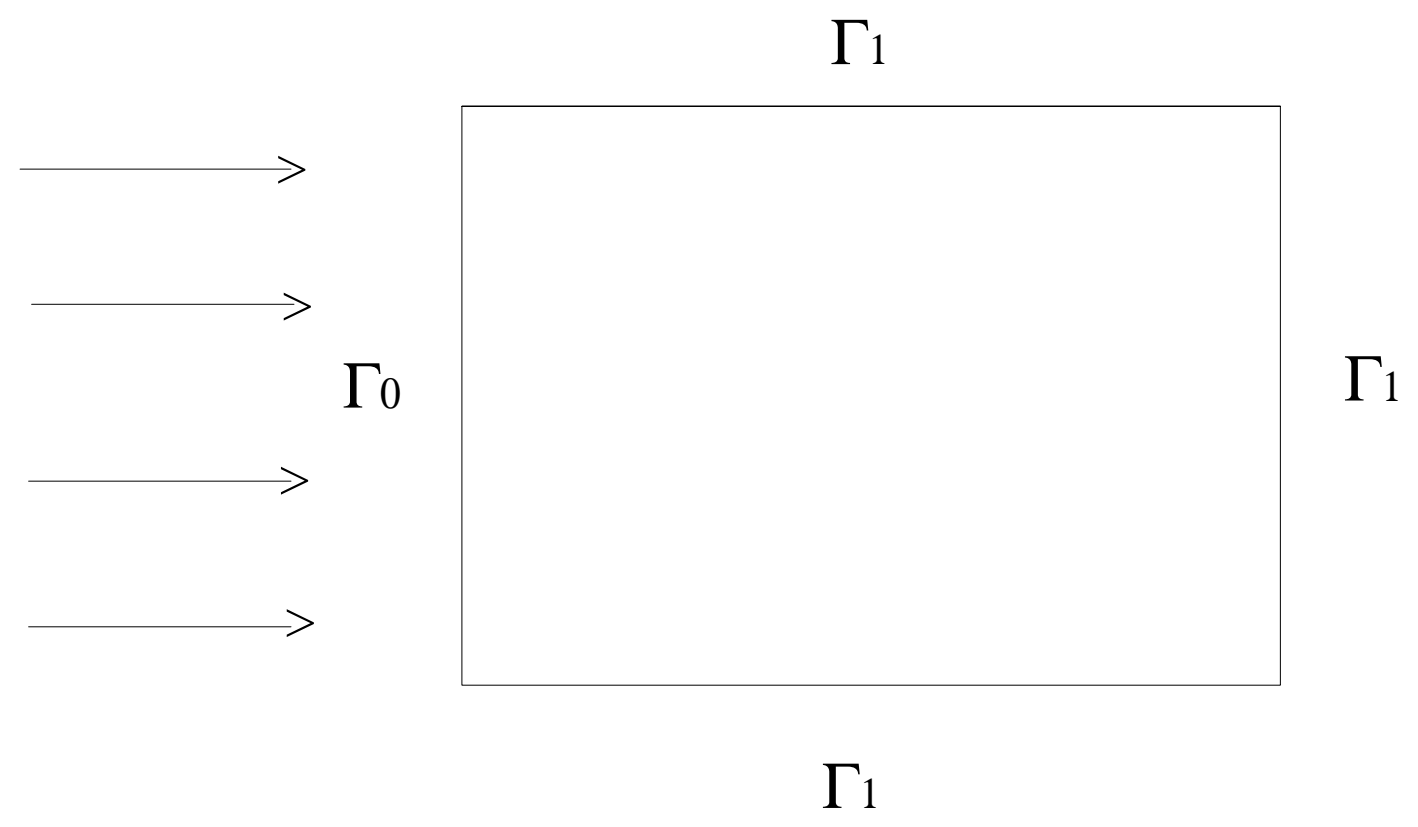

Fig. 3. Sketch of the Boundary

Note that $\gamma(\mu) \frac{\partial \varphi}{\partial n}$ denotes the input flux. The boundary condition on $\Gamma_{0}$ means that the input flux at the boundary $\Gamma_{0}$ is given as a function $f$, determined by the laser source. The other part of the boundary condition means that the energy fluence rate at the boundary $\Gamma_{1}$ is proportional to the output flux, with proportionality constant $\alpha$. This model is by no means perfect, but essentially all of the analysis which follows is applicable with minor changes in boundary conditions. We set this problem in a minimization problem.

\section{Minimization Problem}

Let $F$ be a function from $X$ to $\mathcal{H}^{1}(\Omega)$ defined by $F(\mu)=\varphi$, where $\varphi$ solves (3.4). Let $\tilde{\varphi}$ be given. We assume $\tilde{\varphi}$ has been recovered from measured data by solving (3.3). 
We wish to recover $\mu$ by solving the least-squares minimization problem

$$
\min _{\mu \in X} J(\mu)=\|F(\mu)-\tilde{\varphi}\|_{L^{2}(\Omega)}^{2}
$$

Let $X$ be a subset of $\mathcal{L}^{\infty}(\Omega)$ such that $X=\left\{\mu \in \mathcal{L}^{\infty}(\Omega): 0 \lessgtr \mu_{1} \leq \mu(x) \leq \mu_{2} \lesseqgtr\right.$ $\infty, \mu$ is Lipschitz continuous with $\operatorname{Lip}(\mu) \leq c$ and $\mu=q$ on $\partial \Omega\}$. We assume $\mu$ is the known function $q$ on the boundary, since $\Omega$ is accessible there. The "forward map" $F$ is a function from $X \subset \mathcal{L}^{\infty}(\Omega)$ into $\mathcal{H}^{1}(\Omega)$.

\section{Properties of the Forward Map}

In this section we will study properties of the function $F$. By standard energy estimates [11], $\|F(\mu)\|_{H^{1}(\Omega)} \leq C$ for all $\mu \in X$.

Proposition C.1 $F: X \rightarrow \mathcal{H}^{1}(\Omega)$ is Lipschitz continuous.

Proof. Assume $(\mu+s) \in X$. Let $F(\mu+s)=\varphi+\varphi_{s}$ where $F(\mu)=\varphi$. Then

$$
\begin{aligned}
& -\nabla \cdot \gamma(\mu+s) \nabla\left(\varphi+\varphi_{s}\right)+(\mu+s)\left(\varphi+\varphi_{s}\right)=0, \quad \text { on } \Omega, \\
& -\nabla \cdot \gamma(\mu) \nabla \varphi+\mu \varphi=0, \quad \text { on } \Omega .
\end{aligned}
$$

By subtracting (3.7) from (3.6), we have

$$
-\nabla \cdot \gamma(\mu+s) \nabla \varphi_{s}+(\mu+s) \varphi_{s}+s \varphi-\nabla \cdot(\gamma(\mu+s)-\gamma(\mu)) \nabla \varphi=0 .
$$

Expand $\gamma(\mu+s)$ as $\gamma(\mu+s)=\gamma(\mu)+s D \gamma(\mu)(s)+O\left(\|s\|_{\infty}^{2}\right)$, where $D \gamma(\mu)=-\frac{1}{3\left(\mu_{s}+\mu\right)^{2}}$, the derivative of $\gamma(\mu)$ with respect to $\mu$. By multiplying by $\varphi_{s}$, integrating by parts using $s=0$ on $\partial \Omega$, and the fact that $\|\varphi\|_{H^{1}(\Omega)}$ is uniformly bounded, we find $\left\|\varphi_{s}\right\|_{\mathcal{H}^{1}} \leq$ $C\|s\|_{\infty}$ for some constant $\mathrm{C}$. This shows that $F$ is a Lipschitz continuous function.

Proposition C.2 F is Fréchet differentiable. Furthermore, the Fréchet derivative $D F(\mu): \mathcal{L}^{\infty}(\Omega) \rightarrow \mathcal{L}^{2}(\Omega)$ is a compact operator. 
Proof. From the previous proposition, $\mathrm{F}$ is Lipschitz continuous. Let $F(\mu+s)=$ $\varphi+\varphi_{s}$ as above. If the Fréchet derivative exists, it must equal the Gateaux derivative $D F(\mu)(s)=\lim _{\|s\| \rightarrow 0} \frac{1}{\|s\|} \varphi_{s}:=\psi$ and hence solves

$$
\left\{\begin{array}{l}
-\nabla \cdot \gamma(\mu) \nabla \psi+\mu \psi=\nabla \cdot s D \gamma(\mu) \nabla \varphi-s \varphi \\
\gamma(\mu) \frac{\partial \psi}{\partial n}=0 \text { on } \Gamma_{0}, \\
\gamma(\mu) \frac{\partial \psi}{\partial n}+\alpha \psi=0 \text { on } \Gamma_{1} .
\end{array}\right.
$$

It is obvious that $D F(\mu)(\cdot)$ is linear function on $X$. First we show that $D F(\mu)(\cdot)$ is bounded operator, i.e. $\|\psi\|_{\mathcal{H}^{1}} \leq C\|s\|_{\infty}$ for some constant $C$. From the equation (3.8) by multiplying by $\psi$, it becomes

$$
\int_{\Omega} \gamma(\mu)|\nabla \psi|^{2}+\int_{\Omega} \mu|\psi|^{2}+\int_{\Gamma_{1}} \alpha|\psi|^{2}=\int_{\Omega} \nabla \cdot s D \gamma(\mu) \nabla \varphi \psi-\int_{\Omega} s \varphi \psi
$$

Since $\mu$ and $\mu_{s}$ are bounded below from a positive constant, the left hand side of the equation (3.9) can be bounded below from $c\|\psi\|_{\mathcal{H}^{1}}$. By integrating by parts the right hand side of (3.9) can be bounded above by $c^{\prime}\|s\|_{\infty}$, where $c^{\prime}=c^{\prime}(f)$. Thus we obtain $\|\psi\|_{H^{1}(\Omega)} \leq C\|s\|_{\infty}$. Now, it only remains to show that

$$
\frac{\|F(\mu+s)-F(\mu)-D F(\mu)(s)\|}{\|s\|} \rightarrow 0,
$$

as $\|s\| \rightarrow 0$. By subtracting (3.8) from (3.6), and multiplying by $\left(\varphi_{s}-\psi\right)$, we have

$$
\left\|\varphi_{s}-\psi\right\|_{H^{1}(\Omega)} \leq c\|s\|_{\infty}\left\|\varphi_{s}\right\|_{H^{1}(\Omega)} .
$$

Since $F$ is continuous, $\left\|\varphi_{s}\right\|_{H^{1}(\Omega)} \rightarrow 0$ as $\|s\| \rightarrow 0$. Thus we obtain (3.10). 
Now, we show that $D F(\mu)$ is a compact operator. Let $D F(\mu)\left(\delta_{k}\right)=\psi_{k}$, where $\mu+\delta_{k} \in X$ and $\psi_{k}$ solves

$$
\left\{\begin{array}{l}
\nabla \cdot \gamma(\mu) \nabla \psi_{k}+\mu \psi_{k}=\nabla \cdot \delta_{k} D \gamma(\mu) \nabla \varphi-\delta_{k} \varphi \text { in } \Omega \\
\gamma(\mu) \frac{\partial \psi_{k}}{\partial n}=0, \text { on } \Gamma_{0}, \\
\gamma(\mu) \frac{\partial \psi_{k}}{\partial n}+\alpha \psi_{k}=0, \text { on } \Gamma_{1},
\end{array}\right.
$$

where $\varphi=F(\mu)$ and $\left\|\delta_{k}\right\|_{\infty} \leq C$. Then integrating by parts,

$$
\int_{\Omega} \gamma(\mu)\left|\nabla \psi_{k}\right|^{2}+\int_{\Omega} \mu\left|\psi_{k}\right|^{2}+\alpha \int_{\Gamma_{1}}\left|\psi_{k}\right|^{2}=-\int_{\Omega} \delta_{k} D \gamma(\mu) \nabla \varphi \cdot \nabla \psi_{k}-\int_{\Omega} \delta_{k} \varphi \psi_{k} .
$$

Thus

$$
\begin{aligned}
c_{1}\left\|\psi_{k}\right\|_{H^{1}}^{2} & \leq \int_{\Omega} \gamma(\mu)\left|\nabla \psi_{k}\right|^{2}+\int_{\Omega} \mu\left|\psi_{k}\right|^{2}+\alpha \int_{\gamma_{1}}\left|\psi_{k}\right|^{2}, \\
& =-\int_{\Omega} \delta_{k} D \gamma(\mu) \nabla \varphi \cdot \nabla \psi_{k}-\int_{\Omega} \delta_{k} \varphi \psi_{k}, \\
& \leq\left(\int_{\Omega} D \gamma(\mu)\left|\nabla \varphi \cdot \nabla \psi_{k}\right|+\int_{\Omega}\left|\varphi \psi_{k}\right|\right)\left\|\delta_{k}\right\|_{\infty}, \\
& \leq c_{2}\left(\|\varphi\|_{1}\left\|\psi_{k}\right\|_{1}+\|\varphi\|_{1}\left\|\psi_{k}\right\|_{1}\right)\left\|\delta_{k}\right\|_{\infty}, \\
& =c_{3}\|\varphi\|_{1}\left\|\psi_{k}\right\|_{1}\left\|\delta_{k}\right\|_{\infty} .
\end{aligned}
$$

Thus $\left\{\psi_{k}\right\}$ is a bounded sequence in $\mathcal{H}^{1}(\Omega)$. Since $\mathcal{H}^{1}(\Omega) \rightarrow \mathcal{L}^{2}(\Omega)$ is a compact embedding, $\left\{\psi_{k}\right\}$ has a convergent subsequence in $\mathcal{L}^{2}(\Omega)$.

\section{Compactness of Admissible Solution Space}

Proposition C.3 $X$ is $L^{\infty}$-compact.

Proof. Let $\left\{\mu_{k}\right\} \subseteq X$. Then $\mu_{k} \in W^{1, \infty}(\Omega)$ with $\left\|\mu_{k}\right\|_{\infty} \leq \mu_{2} \neq \infty$. By Theorem 7.10 in [11], there exists a subsequece $\left\{\mu_{k_{j}}\right\}$ which converges to some $\mu \in C^{0}(\Omega)$ with 
respect to $L^{\infty}$-norm. Then,

$$
\begin{aligned}
|\mu(x)-\mu(y)| & \leq\left|\mu(x)-\mu_{k_{j}}(x)\right|+\left|\mu_{k_{j}}(x)-\mu_{k_{j}}(y)\right|+\left|\mu_{k_{j}}(y)-\mu(y)\right| \\
& \leq 2\left\|\mu-\mu_{k_{j}}\right\|_{\infty}+c\|x-y\| \\
& \rightarrow c\|x-y\| \text { as } k_{j} \rightarrow \infty
\end{aligned}
$$

where $c$ is the Lipschitz bound in the definition of $X$. Thus, $\mu \in X$, which shows $X$ is $\mathcal{L}^{\infty}$-compact.

\section{Existence and Uniqueness of Solutions}

Since $X$ is $\mathcal{L}^{\infty}$ - compact (by Proposition C.3) and $F$ is $\mathcal{L}^{\infty}$ continuous(by Proposition C.1), the minimization problem has a solution, by the Weierstrass theorem[29].

Theorem C.1 The minimization problem (3.3) has a solution.

Now we will study the uniqueness of the minimization problem. We will try to prove the uniqueness of solutions result using Taylor expansion. As in the complex plane, a Taylor series converges if the remainder terms $\left|R_{n}\right|=\frac{\left\|D^{n} F(\mu)\right\|}{n !} r^{n} \rightarrow 0$, where $r=\|\nu-\mu\|$. We show that $\left\|D^{n} F(\mu)\right\| \leq c A^{n} n$ ! for some constant $\mathrm{c}$ and A. Thus, we can conclude that $\mathrm{F}$ is analytic in the small neighborhood of $\mu$ with radius less than $\frac{1}{A}$.

The following theorem is proved in [1]

Theorem C.2 Let $X, Y$ be Banach spaces, $U \subset X$ open, $x \in U$. Let $F: U \rightarrow Y$ be p-times differentiable and the derivatives up to the order p-1 continuous. Then for every $\epsilon>0$ there is a $\delta>0$ such that for all $h \in X$ with $\|h\| \leq \delta$,

$$
\left\|F(x+h)-\sum_{j=0}^{p} \frac{1}{j !} D^{j} F(x)(h, . ., h)\right\| \leq \epsilon\|h\|^{p} .
$$


Here, $D^{j} F(x)(h, \ldots, h)$ denotes the $j$-th order derivative of $F$ at $x$, in the direction $h$.

Proposition C.4 $D^{n} F(\mu)\left(s_{1}, s_{2}, . . s_{n}\right)=\varphi^{(n)}$, where $\varphi^{(n)}$ solves

$$
\left\{\begin{array}{l}
-\nabla \cdot \gamma(\mu) \nabla \varphi^{(n)}+\mu \varphi^{(n)}=-s_{n} \varphi^{(n-1)} \\
\quad+\sum_{j=1}^{n} \nabla \cdot s_{j} s_{j+1} \ldots s_{n} D^{(n-j+1)} \gamma(\mu) \nabla \varphi^{(j-1)} \text { in } \Omega, \\
\gamma(\mu) \frac{\partial \varphi^{(n)}}{\partial n}=0, \text { on } \Gamma_{0}, \\
\gamma(\mu) \frac{\partial \varphi^{(n)}}{\partial n}+\alpha \varphi^{(n)}=0, \text { on } \Gamma_{1} .
\end{array}\right.
$$

Proof. The proof is by induction. By Proposition C.2, (3.11) is true for $i=$ 1. Suppose it is true for $i \leq n$ and prove this for $i=n+1$. Let $D^{n} F(\mu+$ $\left.s_{n+1}\right)\left(s_{1}, \ldots, s_{n}\right)=\varphi^{(n)}+\varphi_{\delta}$. i.e.

$$
\begin{aligned}
& -\nabla \cdot\left(\gamma(\mu)+s_{n+1} D \gamma(\mu)+O\left(\left\|s_{n+1}\right\|_{\infty}^{2}\right)\right) \nabla\left(\varphi^{(n)}+\varphi_{s}\right)+\left(\mu+s_{n+1}\right)\left(\varphi^{(n)}+\varphi_{s}\right) \\
& =-s_{n} \varphi^{(n-1)}+\sum_{j=1}^{n} \nabla \cdot s_{j} s_{j+1} \ldots s_{n}\left(D^{(n-j+1)} \gamma(\mu)+s_{n+1} D^{(n-j+2)} \gamma(\mu)\right) \\
& \left.\quad+O\left(\left\|s_{n+1}\right\|_{\infty}^{2}\right)\right) \nabla \varphi^{(j-1)} .
\end{aligned}
$$

Divide by $\left\|s_{n+1}\right\|_{\infty}$ and let $\left\|s_{n+1}\right\|_{\infty} \rightarrow 0$. Then (3.11) is true for $\mathrm{i}=\mathrm{n}+1$.

Proposition C.5 There exists constants $A=A(\mu, \alpha)$ and $c_{0}$ such that

$$
\left\|\varphi^{(n)}\right\|_{\mathcal{H}^{1}(\Omega)} \leq c_{0} A^{n} n !\left\|s_{1}\right\|_{\infty} \ldots\left\|s_{n}\right\|_{\infty}, \text { for all } n
$$

i.e., $\left\|D^{n} F(\mu)\right\| \leq c_{0} A^{n} n$ !.

Proof. Let $c_{n}=\left\|D^{n} F(\mu)\right\|$. The proof is by induction. Obviously, this is true for $\mathrm{i}=0$. Assume that it is true for $i \leq n-1$. We prove it for $i=n$. Let

$$
m=\max _{x \in \Omega} \frac{1}{\mu(x)+\mu_{s}} \leq \frac{1}{\mu_{1}+\mu_{s}} .
$$


Since $D^{n} \gamma(\mu)=(-1)^{n} \frac{n !}{3\left(\mu+\mu_{s}\right)^{n+1}},\left\|D^{n} \gamma(\mu)\right\|_{\infty} \leq \frac{1}{3} m^{n+1} n$ !. Multiply (3.11) by $\varphi^{(n)}$. Integrating by parts, (3.11) becomes

$$
\begin{aligned}
\int_{\Omega} \gamma(\mu)\left|\nabla \varphi^{(n)}\right|^{2}+\int_{\Omega} \mu\left|\varphi^{(n)}\right|^{2} & +\int_{\Gamma_{0}} \alpha|\varphi|^{2} \\
= & -\int_{\Omega} s_{n} \varphi^{(n-1)} \varphi^{(n)} \\
& -\sum_{j=1}^{n} \int_{\Omega} s_{j} \ldots s_{n} D^{n-j+1} \gamma(\mu) \nabla \varphi^{(j-1)} \cdot \nabla \varphi^{(n)} \\
& -\sum_{j=1}^{n} \int_{\Gamma_{0}} s_{j} \ldots s_{n} D^{n-j+1} \gamma(\mu) \frac{\partial \varphi^{(j-1)}}{\partial n} \varphi^{(n)}
\end{aligned}
$$

Then, the left hand side of (3.12) can be bounded below by $\frac{1}{a}\left\|\varphi^{(n)}\right\|_{\mathcal{H}^{1}(\Omega)}^{2}$, where

$$
\frac{1}{a}=\min _{x \in \Omega}\left(\frac{1}{3\left(\mu(x)+\mu_{s}\right)}, \mu(x)\right) \geq \min \left(\frac{1}{3\left(\mu_{2}+\mu_{s}\right)}, \mu_{1}\right)>0 .
$$

On the other hand, if we denote $q_{n}=\left\|s_{1}\right\|_{\infty}\left\|s_{2}\right\|_{\infty} \ldots\left\|s_{n}\right\|_{\infty}$, then (3.12) is bounded above by

$$
\begin{gathered}
c_{n-1} q_{n}\left\|\varphi^{(n)}\right\|_{1}+q_{n} \sum_{j=1}^{n} c_{j-1}\left\|D^{n-j+1} \gamma(\mu)\right\|\left\|\varphi^{(n)}\right\|_{1} \\
+q_{n} \alpha \sum_{j=1}^{n}\left\|\frac{D^{n-j+1} \gamma(\mu)}{\gamma(\mu)}\right\| \beta^{2} c_{j-1}\left\|\varphi^{(n)}\right\|_{1},
\end{gathered}
$$

where $\beta$ is the constant from the trace inequality: $\|f\|_{\mathcal{L}^{2}(\partial \Omega)} \leq \beta\|f\|_{\mathcal{H}^{1}(\Omega)}[11]$. Thus we obtain

$$
\begin{aligned}
\left\|\varphi^{(n)}\right\|_{1} & \leq a\left\{c_{n-1}+\frac{1}{3} \sum_{j=1}^{n} c_{j-1}(n-j+1) ! m^{n-j+2}+\alpha \beta^{2} \sum_{j=1}^{n} c_{j-1}(n-j+1) ! m^{n-j+1}\right\} \\
& =a\left\{c_{n-1}+\frac{1}{3} m \sum_{j=1}^{n} c_{n-j} j ! m^{j}+\alpha \beta^{2} \sum_{j=1}^{n} c_{n-j} j ! m^{j}\right\} .
\end{aligned}
$$

Hence we have the inequality

$$
c_{n} \leq a c_{0}\left\{A^{n-1}(n-1) !+\left(\frac{1}{3} m+\alpha \beta^{2}\right) \sum_{j=1}^{n}(n-j) ! j ! A^{n-j} m^{j}\right\} .
$$


Note that

$$
(n-j) ! j ! \leq n !, \quad \text { for all } j \leq n
$$

We can conclude that

$$
\begin{aligned}
c_{n} & \leq a c_{0}\left(A^{n-1}(n-1) !+\left(\frac{1}{3} m+\alpha \beta^{2}\right) n ! \sum_{j=1}^{n} A^{n-j} m^{j}\right), \\
& =a c_{0}\left(A^{n-1}(n-1) !+\left(\frac{1}{3} m+\alpha \beta^{2}\right) n ! \frac{m\left(A^{n}-m^{n}\right)}{A-m}\right) .
\end{aligned}
$$

Choose A sufficiently large such that $A-m \geq \frac{1}{2} A$. e.g. $A \geq 2 m$. Then (3.13) yields

$$
\begin{aligned}
c_{n} & \leq a c_{0}\left\{A^{n-1}(n-1) !+\left(\frac{1}{3} m+\alpha \beta^{2}\right) n ! \frac{m\left(A^{n}-m^{n}\right)}{\frac{1}{2} A}\right\}, \\
& \leq a c_{0}\left\{A^{n-1}(n-1) !+\left(\frac{1}{3} m+\alpha \beta^{2}\right) n ! \frac{2 m A^{n}}{A}\right\} \\
& =a c_{0}\left\{A^{n-1}(n-1) !+\left(\frac{1}{3} m+\alpha \beta^{2}\right) n ! 2 m A^{n-1}\right\}, \\
& =c_{0} A^{n} n !\left\{\frac{a}{n A}+\frac{2 m^{2} a}{3 A}+\alpha \beta^{2} \frac{2 m a}{A}\right\} .
\end{aligned}
$$

Choose A sufficiently large to make

$$
\left\{\frac{a}{A}+\frac{2 m^{2} a}{3 A}+\alpha \beta^{2} \frac{2 m a}{A}\right\} \leq 1
$$

Then we obtain the desired result $c_{n} \leq c_{0} A^{n} n !$.

Let us consider the case where $F$ is linearized. i.e. $F\left(\mu+\delta_{\mu}\right)=F(\mu)+D F(\mu)\left(\delta_{\mu}\right)$. The following result is proved in [21]. Proving uniqueness for the linearized inverse problem is often the first step to a full uniqueness result [2].

Lemma C.3 Let $\Omega \subset \mathbb{R}^{n}$ be a connected bounded domain. Assume that $u \in \mathcal{C}^{1}(\Omega), v \in$ $\mathcal{C}(\bar{\Omega})$ and $f \in \mathcal{L}^{\infty}(\Omega)$. Assume $\alpha \in \mathcal{L}^{\infty}(\Omega)$ is continuous and differentiable. For given $u$ and $v, \alpha$ satisfies

$$
\nabla u \cdot \nabla \alpha+v \alpha=f \quad \text { in } \Omega
$$


Suppose $|\nabla u| \geq k$ for some positive number $k$ in $\Omega$. Then for any $f$, (3.14) has a unique solution a assuming prescribed values along $\partial \Omega$, and

$$
\|\alpha\|_{\infty} \leq C(u, v) \cdot\left\{\sup _{\partial \Omega}|\alpha|+\frac{[u]}{k^{2}}\|f\|_{\infty}\right\}
$$

where $[u]=\sup _{\Omega} u-\inf _{\Omega} u$.

Proof. We include the proof for completeness. The characteristics of (3.14) are curves of steepest ascent in $u$ given by

$$
\frac{d \mathbf{x}}{d s}=\frac{\nabla u}{|\nabla u|}
$$

where $s$ denotes arclength in the direction of increasing $u$. Since all characteristics have finite length, they must originate and terminate on the boundary.

Let $p \in \Omega$ be an arbitrary interior point. Let $\mathcal{C}$ be the corresponding characteristic through $p$ and $a \in \partial \Omega$, the boundary point at which $\mathcal{C}$ originates. Along $\mathcal{C},(3.14)$ becomes to an ordinary differential equation in $\alpha$ :

$$
|\nabla u| \frac{d \alpha}{d s}+v \alpha=f
$$

Since $|\nabla u| \geq k>0$, we can divide by $|\nabla u|$. Then we have

$$
\frac{d}{d s}\left\{\alpha \exp \left\{-\int_{s}^{0} \frac{v}{|\nabla u|}\right\}\right\}=f \exp \left\{-\int_{s}^{0} \frac{v}{|\nabla u|} d s^{\prime}\right\}
$$

where $s=0$ at $p$. By integrating from $q$ to $p$, we have

$$
\alpha(p)=\alpha(q) \exp \left\{-\int_{-l}^{0} \frac{v}{|\nabla u|} d s^{\prime}\right\}+\int_{-l}^{0} \frac{f}{|\nabla u|} \exp \left\{-\int_{s}^{0} \frac{v}{|\nabla u|} d s^{\prime}\right\} d s
$$

where $l$ is the arclength of $\mathcal{C}$ between $q$ and $p$. Let $r \equiv \sup _{\Omega}\left\{\frac{v}{|\nabla u|}\right\}$. Then we obtain the inequality

$$
|\alpha(p)| \leq|\alpha(q)| \cdot e^{r l}+\frac{\|f\|_{\infty}}{i n f_{\Omega}|\nabla u|} \cdot \frac{e^{r l}-1}{r} .
$$


Note that

$$
|\alpha(q)| \cdot e^{r l} \leq \sup _{\partial \Omega}|\alpha| \cdot \max \left\{1, e^{r l}\right\}
$$

and for any $p \in \Omega$

$$
\frac{e^{r l}-1}{r l} \leq \max \left\{1, e^{r l}\right\} \leq \max \left\{1, e^{r l_{\max }}\right\}
$$

where $l_{\text {max }}$ is the length of the longest characteristic.

By the assumption $\inf _{\Omega}|\nabla u|>0$, a well-defined characteristic passes through each point of $\Omega$. Also, it yields a bound $l_{\max }$ on the length of the longest characteristic,

$$
l_{\max } \leq \frac{[u]}{i n f_{\Omega}|\nabla u|}, \quad[u] \equiv \sup _{\Omega} u-\inf _{\Omega} u
$$

Thus we obtain the desired inequality (3.15).

\section{Lemma C.4 Suppose that}

$$
|\nabla \varphi| \geq k \text { in } \Omega
$$

for some positive number $k$ then the linearized problem $D F(\mu)\left(\delta_{\mu}\right)=\psi$ has a unique solution such that $\mu+\delta_{\mu} \in X$.

Proof. First assume that $\mu=1$. Let $1+\delta_{\mu} \in X$. Then (3.8) becomes

$$
-\gamma(1) \triangle \psi+\psi=D \gamma(1) \nabla \cdot \delta_{\mu} \nabla \varphi-\delta_{\mu} \varphi, \text { in } \Omega
$$

We want to show that the kernel of the Fréchet derivative at $\mu=1$ is trivial. That is, show that $D F(1)\left(\delta_{\mu}\right)=0$ implies $\delta_{\mu}=0$. Suppose $D F(1)\left(\delta_{\mu}\right)=0$. Then we have

$$
\begin{aligned}
0 & =D \gamma(1) \nabla \cdot \delta_{\mu} \nabla \varphi-\delta_{\mu} \varphi \\
& =D \gamma(1) \nabla \delta_{\mu} \cdot \nabla \varphi+D \gamma(1) \delta_{\mu} \Delta \varphi-\delta_{\mu} \varphi
\end{aligned}
$$


Thus we have

$$
\nabla \delta_{\mu} \cdot \nabla \varphi+\delta_{\mu}\left(\triangle \varphi-\frac{1}{D \gamma(1)} \varphi\right)=0
$$

By the previous lemma, we could obtain $\delta_{\mu} \equiv 0$, since $\delta_{\mu}=0$ on the boundary.

Now we show the same result for an arbitrary $\mu \in X$. For the arbitrary $\mu \in X$ (3.8) becomes

$$
-\nabla \cdot \gamma(\mu) \nabla \psi+\mu \psi=\nabla \cdot D \gamma(\mu) \delta_{\mu} \nabla \varphi-\delta_{\mu} \varphi
$$

for $\mu+\delta_{\mu} \in X$. If $\psi=0$ then

$$
\begin{aligned}
0 & =\nabla \cdot D \gamma(\mu) \delta_{\mu} \nabla \varphi-\delta_{\mu} \varphi \\
& =\nabla D \gamma(\mu) \delta_{\mu} \cdot \nabla \varphi-\delta_{\mu} \varphi \\
& =D \gamma(\mu) \nabla \delta_{\mu} \cdot \nabla \varphi+\delta_{\mu}\{\nabla D \gamma(\mu) \cdot \nabla \varphi+D \gamma(\mu) \triangle \varphi-\varphi\}
\end{aligned}
$$

Since $D \gamma(\mu)$ is below from zero, we could divide (3.17) by $D \gamma(\mu)$. Then

$$
\nabla \delta_{\mu} \cdot \nabla \varphi+\delta_{\mu} P=0
$$

where $P$ is

$$
P=\frac{\nabla D \gamma(\mu)}{D \gamma(\mu)} \cdot \nabla \varphi+\triangle \varphi-\frac{\varphi}{D \gamma(\mu)}
$$

Then similarly we have by Lemma C.3 that $\delta_{\mu} \equiv 0$ since $\delta_{\mu}=0$ on the boundary. $\square$

To get a local uniqueness result, we wish to show if $F\left(\mu+\delta_{\mu}\right)=F(\mu)$ for $\mu+\delta_{\mu} \in X$, then $\delta_{\mu}=0$, for sufficiently small $\left\|\delta_{\mu}\right\|$. Since $F$ is analytic in a small neighborhood, it suffices to show if $D F(\mu)\left(\delta_{\mu}\right)+\frac{1}{2 !} D^{2} F(\mu)\left(\delta_{\mu}, \delta_{\mu}\right)+\ldots=0$, then $\delta_{\mu}=0$. First, we show if

$$
D F(\mu)\left(\delta_{\mu}\right)+\frac{1}{2 !} D^{2} F(\mu)\left(\delta_{\mu}, \delta_{\mu}\right)+\ldots+\frac{1}{n !} D^{n} F(\mu)\left(\delta_{\mu}, \delta_{\mu}, \ldots, \delta_{\mu}\right)=0
$$

for any $\mathrm{n}$, then $\delta_{\mu}=0$. 
Lemma C.5 Let $n$ be given. Let $F_{2}$ be such that

$$
F_{2}\left(\varphi, \ldots \varphi^{(n-1)}\right)=D \gamma(\mu) \sum_{i=1}^{n} \frac{1}{i !} \varphi^{(i-1)}
$$

Suppose $\left|\nabla F_{2}\right| \geq k$, for some positive number $k$. Suppose (3.18) is true. Then $\delta_{\mu} \equiv 0$ provided $\mu+\delta_{\mu} \in X$.

Proof. First assume that $\mu \equiv 1$. From Proposition C.4, we get

$$
\begin{aligned}
0= & -s \sum_{i=1}^{n} \frac{1}{i !} \varphi^{(i-1)}+\sum_{i=1}^{n} \frac{1}{i !} \sum_{j=1}^{i} \nabla \cdot s^{j} D^{j} \gamma(1) \nabla \varphi^{(i-j)} \\
= & -s \sum_{i=1}^{n} \frac{1}{i !} \varphi^{(i-1)}+\sum_{i=1}^{n} \frac{1}{i !} \sum_{j=2}^{i} D^{j} \gamma(1)\left(s^{j} \triangle \varphi^{(i-j)}+j s^{j-1} \nabla s \cdot \nabla \varphi^{(i-j)}\right) \\
& +\sum_{i=1}^{n} \frac{1}{i !}\left(D \gamma(1) \nabla s \cdot \nabla \varphi^{(i-1)}+D \gamma(1) s \triangle \varphi^{(i-1)}\right) \\
= & s F_{1}+\nabla s \cdot \nabla F_{2},
\end{aligned}
$$

where

$$
\begin{aligned}
F_{1}= & F_{1}\left(s, \varphi, \ldots \varphi^{(n-1)}\right) \\
= & -\sum_{i=1}^{n} \frac{1}{i !} \varphi^{(i-1)}+\sum_{i=1}^{n} \frac{1}{i !}\left\{\sum_{j=2}^{i} D^{j} \gamma(1)\left(s^{j-1} \triangle \varphi^{(i-j)}+j s^{j-2} \nabla s \cdot \nabla \varphi^{(i-j)}\right)\right. \\
& \left.+D \gamma(1) \triangle \varphi^{(i-1)}\right\} .
\end{aligned}
$$

The result follows immediately by applying Lemma C.3 to (3.19).

Now let's prove it for arbitrary $\mu \in X$. We have

$$
\begin{aligned}
0 & =-s \sum_{i=1}^{n} \frac{1}{i !} \varphi^{(i-1)}+\sum_{i=1}^{n} \frac{1}{i !} \sum_{j=1}^{i} \nabla \cdot s^{j} D^{j} \gamma(\mu) \nabla \varphi^{(i-j)} \\
& =s F_{1}+\nabla s \cdot \nabla F_{2}
\end{aligned}
$$


where

$$
\begin{aligned}
F_{1}= & \sum_{i=1}^{n} \frac{1}{i !} \varphi^{(i-1)}+\sum_{i=1}^{n} \frac{1}{i !} \sum_{j=1}^{i} s^{j-1} \nabla \cdot D^{j} \gamma(\mu) \nabla \varphi^{(i-j)} \\
& +\sum_{i=2}^{n} \frac{1}{i !} \sum_{j=2}^{i} D^{j} \gamma(\mu) j \nabla s^{j-1} \cdot \nabla \varphi^{(i-j)} \\
F_{2}= & \sum_{i=1}^{n} \frac{1}{i !} D \gamma(\mu) \varphi^{i-1} .
\end{aligned}
$$

Even though $F_{1}$ depends on $s$, estimate (3.15) implies $s=0$ since $\sup _{\partial \Omega}|s|=\|f\|_{\infty}=$ 0 .

Theorem C.6 Let $F_{2}$ be such that

$$
F_{2}\left(\varphi, \ldots \varphi^{(n)}, \ldots\right)=D \gamma(\mu) \sum_{i=1}^{\infty} \frac{1}{i !} \varphi^{(i-1)} .
$$

Suppose $\left|\nabla F_{2}\right| \geq k$, for some positive number $k$. Then the minimization problem (3.5) has a locally unique solution.

Proof. Suppose that $F(\mu)=F(\mu+s)$ for $\mu, \mu+s \in X$. Assume $\|s\|_{\infty}$ is sufficiently small that the Taylor series converges. Then

$$
D F(\mu)+\ldots+\frac{1}{n !} D^{n} F(\mu)+\ldots=0,
$$

Multiplying (3.11) by $\frac{1}{n !}$ and summing from $n=1$ to $\infty$, we have

$$
\sum_{n=1}^{\infty} \frac{1}{n !}\left\{-s \varphi^{(n-1)}+\sum_{j=1}^{n} \nabla \cdot s^{j} D^{j} \gamma(\mu) \nabla \varphi^{(n-j)}\right\}=0 .
$$


We can write (3.21) as

$$
\begin{aligned}
0= & \sum_{n=1}^{\infty} \frac{1}{n !}\left\{-s \varphi^{(n-1)}+\sum_{j=1}^{n} \nabla \cdot s^{j} D^{j} \gamma(\mu) \nabla \varphi^{(n-j)}\right\} \\
= & -\sum_{n=1}^{\infty} \frac{1}{n !} s \varphi^{(n-1)}+\sum_{n=1}^{\infty} \frac{1}{n !} \sum_{j=1}^{n} \nabla \cdot s^{j} D^{j} \gamma(\mu) \nabla \varphi^{(n-j)} \\
= & -\sum_{n=1}^{\infty} \frac{1}{n !} s \varphi^{(n-1)}+\sum_{n=1}^{\infty} \frac{1}{n !} \sum_{j=1}^{n}\left\{s^{j} \nabla \cdot D^{j} \gamma(\mu) \nabla \varphi^{(n-j)}\right. \\
& \left.+D^{j} \gamma(\mu) \nabla s^{j} \cdot \nabla \varphi^{(n-j)}\right\} \\
= & -\sum_{n=1}^{\infty} \frac{1}{n !} s \varphi^{(n-1)}+\sum_{n=2}^{\infty} \frac{1}{n !} \sum_{j=2}^{n}\left\{s^{j} \nabla \cdot D^{j} \gamma(\mu) \nabla \varphi^{(n-j)}\right. \\
& \left.+D^{j} \gamma(\mu) \nabla s^{j} \cdot \nabla \varphi^{(n-j)}\right\}+\sum_{n=1}^{\infty} \frac{1}{n !} D \gamma(\mu) \nabla s \cdot \nabla \varphi^{(n-1)} \\
= & s F_{1}+\nabla s \cdot \nabla F_{2},
\end{aligned}
$$

where

$$
\begin{aligned}
F_{1}= & -\sum_{n=1}^{\infty} \frac{1}{n !} \varphi^{(n-1)}+\sum_{n=2}^{\infty} \frac{1}{n !} \sum_{j=2}^{n}\left\{s^{j-1} \nabla \cdot D^{j} \gamma(\mu) \nabla \varphi^{(n-j)}\right. \\
& \left.+j s^{j-2} D^{j} \gamma(\mu) \nabla s \cdot \nabla \varphi^{(n-j)}\right\} \\
F_{2}= & \sum_{n=1}^{\infty} \frac{1}{n !} D \gamma(\mu) \varphi^{(n-1)} .
\end{aligned}
$$

By Lemma C.3 we obtain the local uniqueness result. 


\section{CHAPTER IV}

\section{NUMERICAL RESULTS}

As a computational strategy for solving the reconstruction problem, we used the finite element method (FEM). In all our numerical experiments we consider a domain of the size $1 \times 1 \mathrm{~cm}^{2}$ which is uniformly divided into $64 \times 64$ cells on a fixed grid. We assume that the absorption coefficient $\mu$ is piecewise constant with positive upper and lower bounds 2 and 1 respectively. We set the scattering coefficient $\mu_{s}$ as a constant 4 .

The discretized minimization problem (3.5) is approached using two different strategies: the gradient descent method, and the levelset method.

\section{A. Gradient Descent Method}

We used the gradient descent method in two ways. The first section shows the result of the regular gradient descent method. The next section describes the gradient descent method with total variation regularization.

\section{Gradient Descent}

Proposition A.1 Let $J(\mu)$ be defined as in (3.5). There exists the Fréchet derivative $D J(\mu)$ of $J: X \rightarrow \mathbb{R}$. Furthermore $D J(\mu) \in \mathcal{L}^{2}(\Omega)$, for all $\mu$.

Proof. It was established in the previous chapter that $F: X \rightarrow \mathcal{H}^{1}(\Omega)$ is Fréchet differentiable. Since the squared $\mathcal{L}^{2}$ norm is differentiable on $\mathcal{H}^{1}$, it follows that $J(\mu)=\|F(\mu)-\tilde{\varphi}\|_{L^{2}(\Omega)}^{2}$ is Fréchet differentiable. We now compute the Fréchet 
derivative of $J(\mu)$.

$$
\begin{aligned}
J\left(\mu+\delta_{\mu}\right)-J(\mu)= & \left\|F\left(\mu+\delta_{\mu}\right)-\tilde{\varphi}\right\|^{2}-\|F(\mu)-\tilde{\varphi}\|^{2} \\
= & <F\left(\mu+\delta_{\mu}\right)-\tilde{\varphi}, F\left(\mu+\delta_{\mu}\right)-F(\mu)> \\
& +<F(\mu)-\tilde{\varphi}, F\left(\mu+\delta_{\mu}\right)-F(\mu)>.
\end{aligned}
$$

Divide by $\delta_{\mu}$ and let $\left\|\delta_{\mu}\right\|_{\infty}$ approach 0 . Then,

$$
\begin{aligned}
D J(\mu)\left(\delta_{\mu}\right) & =2<D F(\mu)\left(\delta_{\mu}\right), F(\mu)-\tilde{\varphi}>, \\
& =2<\delta_{\mu}, D F^{*}(F(\mu)-\tilde{\varphi})>
\end{aligned}
$$

Thus we can identify the operator $D J(\mu)(\cdot)$ with the "gradient" function $D J(\mu)=$ $2 D F(\mu)^{*}(F(\mu)-\tilde{\varphi})$. Let $D F(\mu)\left(\delta_{\mu}\right)=\psi$ where $\psi$ solves $(3.8)$. Then, $\frac{1}{2} D J(\mu)\left(\delta_{\mu}\right)=$ $\int_{\Omega} \psi(\varphi-\tilde{\varphi})=\int_{\Omega} \psi g$ by setting $g=\varphi-\tilde{\varphi}$. Let $u \in \mathcal{H}^{1}(\Omega)$ solve

$$
\left\{\begin{array}{l}
-\nabla \cdot \gamma(\mu) \nabla u+\mu u=g \text { in } \Omega \\
\gamma(\mu) \frac{\partial u}{\partial n}=f \text { on } \Gamma_{0}, \\
\gamma(\mu) \frac{\partial u}{\partial n}+\alpha u=0 \text { on } \Gamma_{1} .
\end{array}\right.
$$

Then using (3.8),

$$
\begin{aligned}
\frac{1}{2} D J(\mu)\left(\delta_{\mu}\right) & =\int_{\Omega} \psi g \\
& =\int_{\Omega} \psi(-\nabla \cdot \gamma(\mu) \nabla u+\mu u) \\
& =\int_{\Omega} u(-\nabla \cdot \gamma(\mu) \nabla \psi+\mu \psi) \\
& =\int_{\Omega}\left(\nabla \cdot \delta_{\mu} D \gamma(\mu) \nabla \varphi-\delta_{\mu} \varphi\right) \\
& =-\int_{\Omega} \delta_{\mu}(u \varphi-D \gamma(\mu) \nabla \varphi \cdot \nabla u) \\
& =<\delta_{\mu},-(u \varphi+D \gamma(\mu) \nabla \varphi \cdot \nabla u)>
\end{aligned}
$$


Combine with (4.1). Then we have

$$
\begin{aligned}
D J(\mu) & =2 D F(\mu)^{*}(F(\mu)-\tilde{\varphi}) \\
& =-2(u \varphi+D \gamma(\mu) \nabla \varphi \cdot \nabla u) .
\end{aligned}
$$

By Theorem 8.8 in [11], $u, \varphi \in W^{2,2}(\Omega)$. Thus $u, \varphi, \nabla u, \nabla \varphi \in W^{1,2}(\Omega)$. By Theorem 7.26 in [11], there is a compact embedding from $W^{1,2}(\Omega)$ to $\mathcal{L}^{4}(\Omega)$. Thus $u \varphi$ and $\nabla u \cdot \nabla \varphi$ are in $\mathcal{L}^{2}(\Omega)$ and we can conclude that $D J(\mu) \in \mathcal{L}^{2}(\Omega)$.

Note that by using (4.3), the gradient $D J(\mu)$ can be calculated with only two solutions of the diffusion equation.

Our basic implementation of the gradient descent method is :

1. Choose initial guess $\mu_{0}$ and initial stepsize $c$. Set $\mathrm{j}=0$.

Define $\delta J_{j}=D J\left(\mu_{j}\right)$.

2. While $\left\|\delta J_{j}\right\|>$ tol, do

(a) If $J\left(\mu_{j}-c * \delta J_{j}\right)<J\left(\mu_{j}\right)$, then

$$
\mu_{j+1}=\mu_{j}-c * \delta J_{j}
$$

else,

$$
c=c / 2
$$

end

(b) Set $j=j+1$, compute $\delta J_{j}=D J\left(\mu_{j}\right)$ from equation (4.3).

Figure 4(b) shows a typical reconstruction result from the gradient descent method, using the initial guess $\mu_{0} \equiv 1$. The original solution of this reconstruction is shown in Figure 4(a). Even though the original solution has maximum value 2 , the reconstruction result has maximum value approximately 1.5 . This is because the derivative of $J, D J(\mu)=2 D F^{*}(F(\mu)-\tilde{\varphi})$, where $D F$ and hence $D F^{*}$ are com- 


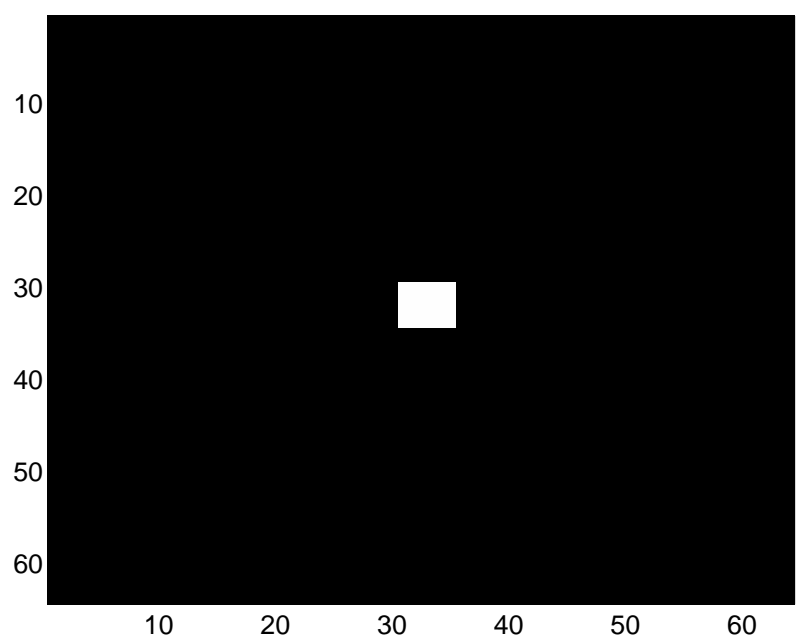

(a) Real Solution

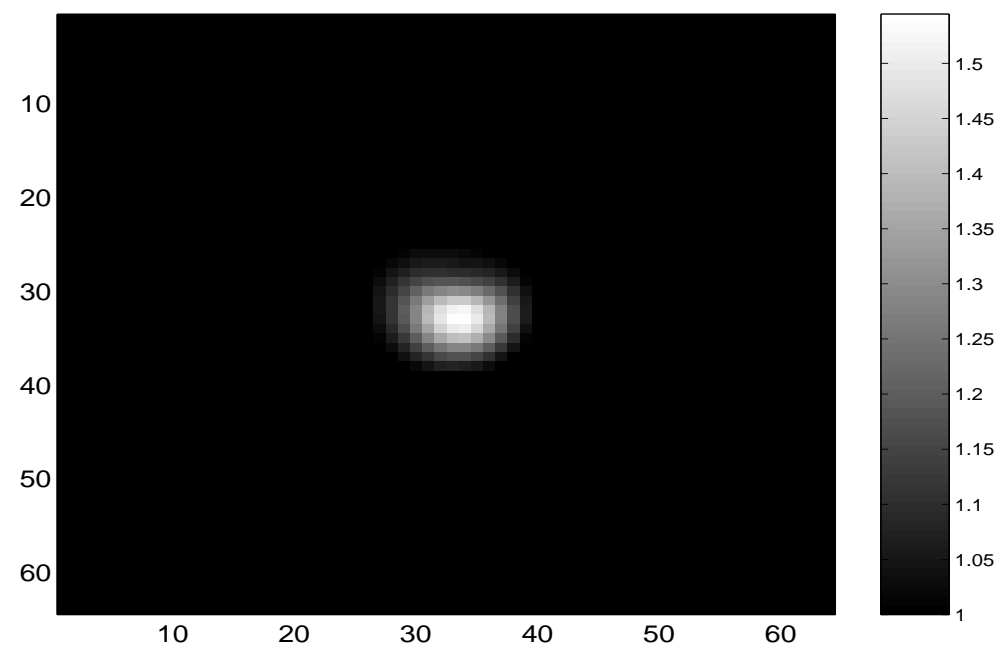

(b) Gradient Decent Method

Fig. 4. Result of the Gradient Descent Method 
pact operators. Thus most of the eigenvalues are close to zero, and the gradients tend to be overly smoothed.

As in Figure 4(b), this method reconstructs the "blocky" model into a blurry model. Here "blocky" means that the conductivity is a piecewise constant function for which the set of discontinuities has relatively small measure in codimension 1 [4].

\section{Minimal Total Variation Regularization}

One way to reduce the blurring effect is an "image enhancement" technique which was applied to the linearized electrical impedance tomography problem by Dobson and Santosa $[4,6]$. The technique is essentially a nonlinear regularization by total variation, following ideas introduced by Rudin, Osher, and Fatemi for image denoising [23]. Measuring the total variation has long been recognized as an effective way to quantify the "simplicity" of a given signal or function. It measures the oscillations of a given function, but still admits discontinuities. This technique has been used in various applications such as image reconstruction, inverse problems, optimal design, and electrical impedance tomography [4, 23].

Consider

$$
\min _{\mu \in X} \tilde{J}(\mu)=\|F(\mu)-\tilde{\varphi}\|_{L^{2}(\Omega)}^{2}+\beta \int_{\Omega}|\nabla \mu|,
$$

where $\nabla \mu$ denotes the gradient of $\mu$ in the sense of distributions; $\nabla \mu$ is a vector valued Radon measure and

$$
T V(\mu)=\int_{\Omega}|\nabla \mu|
$$

is the total variation of $\mu$. We denote by $B V(\Omega)$ the space of functions of bounded variation with the norm

$$
\|\mu\|_{B V(\Omega)} \equiv\|\mu\|_{L^{1}(\Omega)}+T V(\mu) .
$$


We see that $\mu \in X \subset B V(\Omega)$. The quantity $\beta$ is a positive number, called the regularization parameter.

The main computational difficulty is due to the nondifferentiablility of the total variation functional, which precludes direct application of derivative-based minimization methods. For this reason, we modify the total variation functional with a small smoothing parameter and solve the resulting problem. The problem can be written

$$
\min _{\mu \in X} J_{\epsilon}(\mu)=\|F(\mu)-\tilde{\varphi}\|_{L^{2}(\Omega)}^{2}+\beta \int_{\Omega} h_{\epsilon}(|\nabla \mu|)
$$

where

$$
h_{\epsilon}(s)= \begin{cases}s & \text { if } s>\epsilon \\ \frac{s^{2}}{2 \epsilon}+\frac{\epsilon}{2} & \text { if } s \leq \epsilon\end{cases}
$$

Then $h_{\epsilon}$ is $\mathcal{C}^{1}(\Omega)$ for $\epsilon>0$. We call $\epsilon$ a smoothing parameter. The function $h_{\epsilon}$ rounds off the corner in the absolute value.

In our numerical experiments, we discretize the absorption coefficient $\mu$ to lie in the space of piecewise constant functions on a uniform grid with $m \times n$ cells. In this case $h_{\epsilon}(|\nabla \mu|)$ is a measure supported on the lines of the grid. If we denote $\mu_{(i, j)}$ the value of $\mu$ on the $(i, j)$-th cell, then we have

$$
\int_{\Omega} h_{\epsilon}(|\nabla \mu|)=\frac{1}{n m} \sum_{i=1}^{m-1} \sum_{j=1}^{n-1} h_{\epsilon}\left(\left|\mu_{(i+1, j)}-\mu_{(i, j)}\right|\right)+h_{\epsilon}\left(\left|\mu_{(i, j+1)}-\mu_{(i, j)}\right|\right) .
$$

The (i,j)-th gradient, for $1<i<m, 1<j<n$, of the functional $J_{0}:=\int_{\Omega} h_{\epsilon}(|\nabla \mu|)$ is [4]

$$
\begin{aligned}
\frac{\partial J_{0}}{\partial \mu_{(i, j)}} & =q_{\epsilon}\left(\left|\mu_{(i, j)}-\mu_{(i-1, j)}\right|\right)\left(\mu_{(i, j)}-\mu_{(i-1, j)}\right)-q_{\epsilon}\left(\left|\mu_{(i+1, j)}-\mu_{(i, j)}\right|\right)\left(\mu_{(i+1, j)}-\mu_{(i, j)}\right) \\
& +q_{\epsilon}\left(\left|\mu_{(i, j)}-\mu_{(i, j-1)}\right|\right)\left(\mu_{(i, j)}-\mu_{(i, j-1)}\right)-q_{\epsilon}\left(\left|\mu_{(i, j+1)}-\mu_{(i, j)}\right|\right)\left(\mu_{(i, j+1)}-\mu_{(i, j)}\right)
\end{aligned}
$$




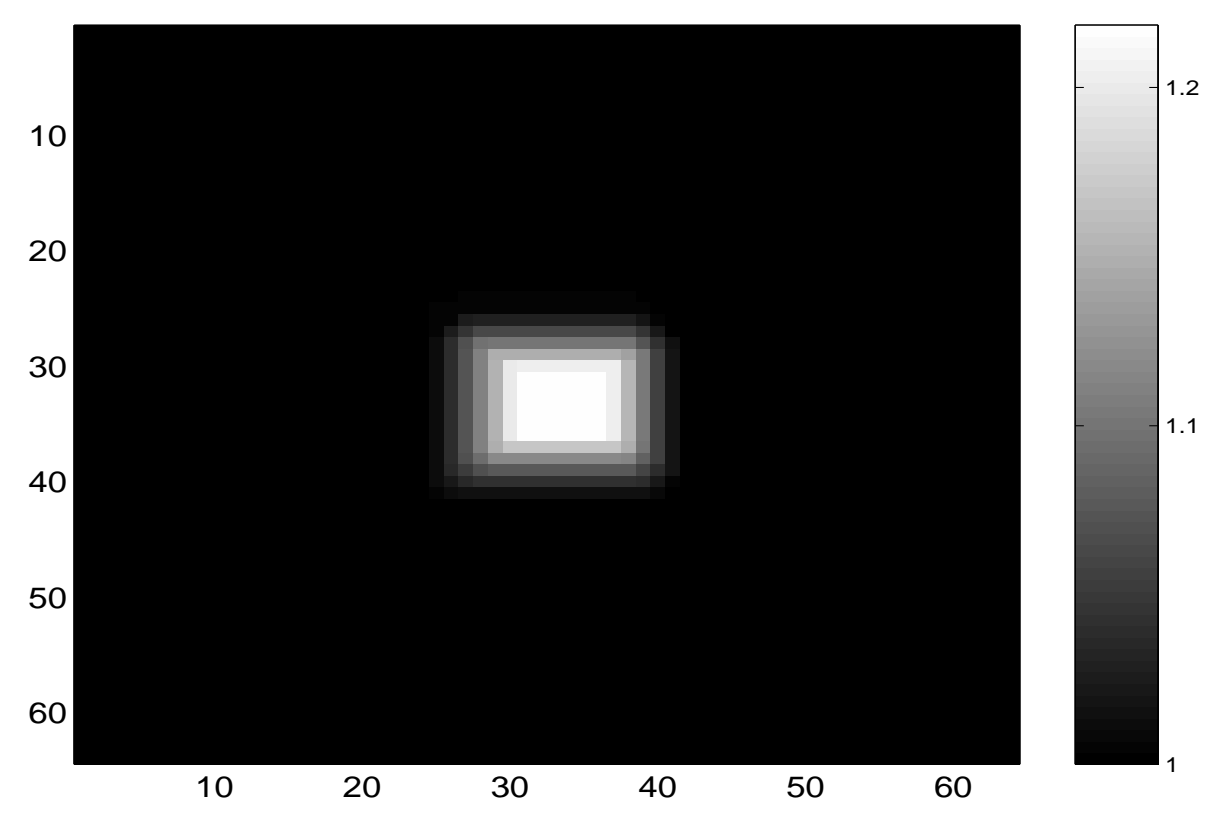

Fig. 5. Blocky Model

where

$$
q_{\epsilon}(s)=\frac{h_{\epsilon}^{\prime}(s)}{s}= \begin{cases}\frac{1}{s} & \text { if } s>\epsilon \\ \frac{1}{\epsilon} & \text { if } s \leq \epsilon\end{cases}
$$

and $\frac{\partial J_{0}}{\partial \mu_{(i, j)}}=0$ if $i=1$ or $m, j=1$ or $n$.

For the same exact solution and starting guess in Figure 4, Figure 5 illustrates the result of the total variation regularized method, with the regularization parameter $\beta=5 \times 10^{-5}$ and the smoothing parameter $\epsilon=10^{-4}$. It is "more blocky" than the unregularized method, but it has fat block compared to the real solution. Since the total variation term also favors small function values, the maximum value of the reconstruction is too small (approximately 1.25). 


\section{B. Levelset Method}

The main goal of this problem is to get an accurate reconstruction of optical absorption in biological tissue. That is, to separate diseased tissue from the healthy tissue. If we assume that the absorption coefficient is piecewise constant, e.g., that $\mu=\mu_{h}$ indicates healthy tissue and $\mu=\mu_{d}$ indicates diseased tissue, it could be reconstructed by using levelset method.

The levelset approach was first given by Osher and Sethian for describing the motion of curves and surfaces [20]. Recently, the levelset method has been used in various problems, for example image enhancement, computer vision, interface problems, crystal growth, or etching and deposition in the microchip fabrication [13, 18]. Santosa used the levelset representation as a part of a solution scheme for inverse problems involving obstacles [24]. More recently, this method is applied to a reconstruction for diffuse optical tomography by Dorn [9]. The differences between [9] and the present work are that Dorn does not consider the ultrasound modulation, and he uses the Boltzmann equation rather than the diffusion approximation. Also, his algorithm is to find the absorption and scattering coefficients simultaneously. Our model is to find only the absorption coefficient for a given scattering coefficient. This method has a powerful reconstruction result in this problem.

As we did in the previous chapter in section 2, we add the total variation regularization term in the levelset method. To our knowledge, this is the first time total variation has been used to regularize a levelset method. 


\section{Levelset}

Suppose the measured data is given. Assume that the solution function has two values in the region $\Omega$. The goal of this problem is find the region $\mathrm{D}$ in the equation

$$
F(\mu)=\varphi
$$

where

$$
\mu(x)=\left\{\begin{array}{l}
\mu_{d} \text { for } x \in D, \\
\mu_{h} \text { for } x \notin D .
\end{array}\right.
$$

The levelset approach is fast and accurate compared to the gradient descent method. It attempts to reconstruct a two-valued piecewise constant function, rather than a general $L^{\infty}$ function. In the levelset method, the region $D$ is represented by a continuous function, called a levelset function. There are many advantages to represent the unknown region through a levelset function. We don't need to make $a$ priori assumptions about the connectedness and the nature of D [24]. In the levelset approach, we generate a sequence of functions $\eta_{k}$ such that $D_{k} \rightarrow D$, where $\partial D_{k}=$ $\left\{x: \eta_{k}(x)=0\right\}$. The function $\mu$ in (4.4) can be represented through a levelset function as follows.

$$
\mu(x)=\left\{\begin{array}{l}
\mu_{d} \text { for }\{x: \eta(x)<0\} \\
\mu_{h} \text { for }\{x: \eta(x)>0\} .
\end{array}\right.
$$

Under this description, problem (4.4) becomes:

Find $\eta(x)$ in the equation (4.5) such that $F(\mu)=\varphi$.

We need to calculate the variation of $\mu$ caused by variation in $\eta$. To do this calculation, let $x$ be a point on the curve $\eta(x)=0$. Assume that $\eta(x)$ is perturbed 
by a small variation $\delta \eta(x)$. Let $\delta x$ be the resulting variation of the point $x$. That is,

$$
(\eta+\delta \eta)(x+\delta x)=0 .
$$

Let $D^{\prime}$ be the resulting variation in the region, which is the set of $x$ which satisfies (4.6). By expanding (4.6) by Taylor series, we obtain

$$
\delta \eta+\nabla \eta \cdot \delta x=0
$$

By using (4.7), we find the update for $\mu$ as [24]

$$
\delta \mu=\left.\left(\mu_{d}-\mu_{h}\right) \frac{\nabla \eta}{|\nabla \eta|} \cdot \delta x\right|_{x \in \partial D} .
$$

The algorithm for the levelset method as proposed in [24] is,

1. Choose $\eta_{0}(x) ;$ Set $\mathrm{j}=0$.

2. Compute associated $\mu$;

$$
\mu(x)= \begin{cases}\mu_{h} & \text { if } \eta_{0}(x)>0, \\ \mu_{d} & \text { if } \eta_{0}(x)<0 .\end{cases}
$$

if $J(\mu)>$ tol, do

(a) Solve $D F(\mu)^{*} D F(\mu)\left(\delta_{\mu}\right)=D F(\mu)^{*}(\tilde{\varphi}-F(\mu))=-\frac{1}{2} D J(\mu)$.

(b) Set $\delta \eta(x)=-\frac{\delta_{\mu}}{\mu_{d}-\mu_{h}}\left|\nabla \eta_{j}(x)\right|$

(c) Set $\mathrm{j}=\mathrm{j}+1$, update $\eta_{j+1}=\eta_{j}+\delta \eta$.

In the numerical experiments, we assume the absorption coefficient $\mu$ is piecewise constant on each grid cell while the discrete levelset function $\eta$ is piecewise bilinear. This leads to the problem of how to approximate $\mu$ given the update formula (4.5), as illustrated in Figure 6. 


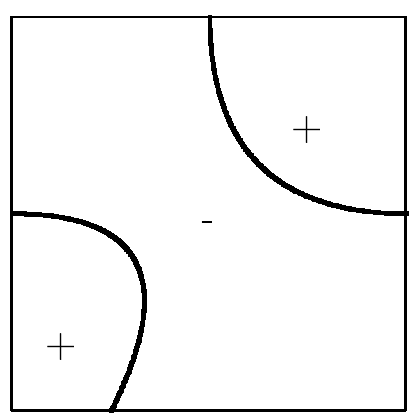

Fig. 6. Volume Fraction

For simplicity, we calculate a volume fraction over each cell $\Omega_{j}$ :

$$
V_{j}=\frac{|\{x: \eta(x)>0\}|}{\left|\Omega_{j}\right|},
$$

and set

$$
\mu_{j}= \begin{cases}\mu_{h} & \text { if } V_{j}>0.5 \\ \mu_{d} & \text { if } V_{j} \leq 0.5\end{cases}
$$

Also, in step (b), we approximate the model value of $\delta \eta$ by averaging over neighboring cells.

The levelset method for this problem is fast and accurate compared with the gradient descent method. This method typically converges to the actual solution within 30 iterations while the gradient descent method needs thousands of iterations to get a small tolerance. Figure 7 shows the reconstruction of the shape of the letter A. We start with the Gaussian function as an initial guess for the levelset function. Figure 8 and Figure 9 show the initial and the final levelset functions for the A-shape example. It has an exact reconstruction with 22 iterations. 

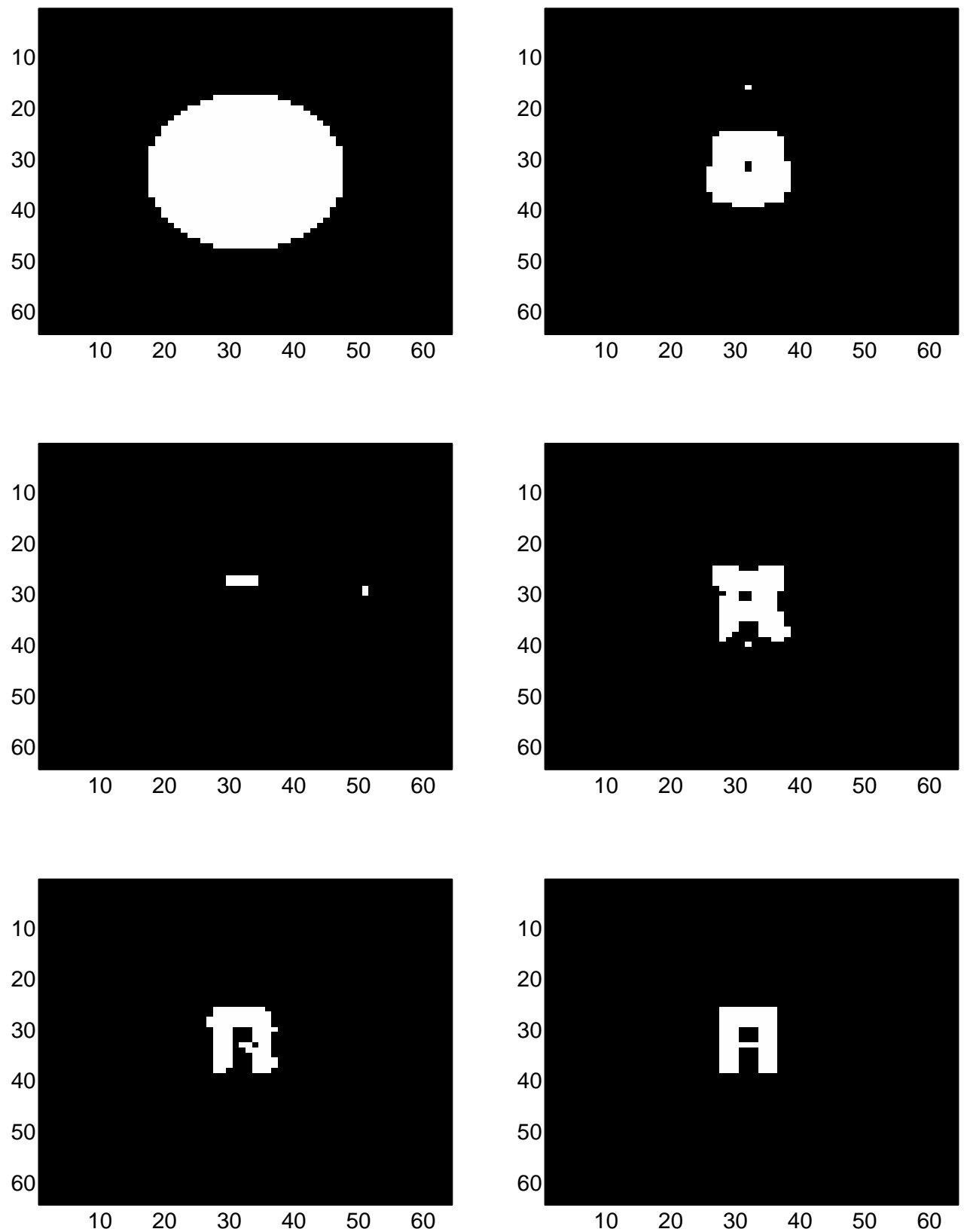

Fig. 7. Reconstruction of A-shaped Block.

(using levelset approach at 1, 3, 4, 8, 12, 22) 


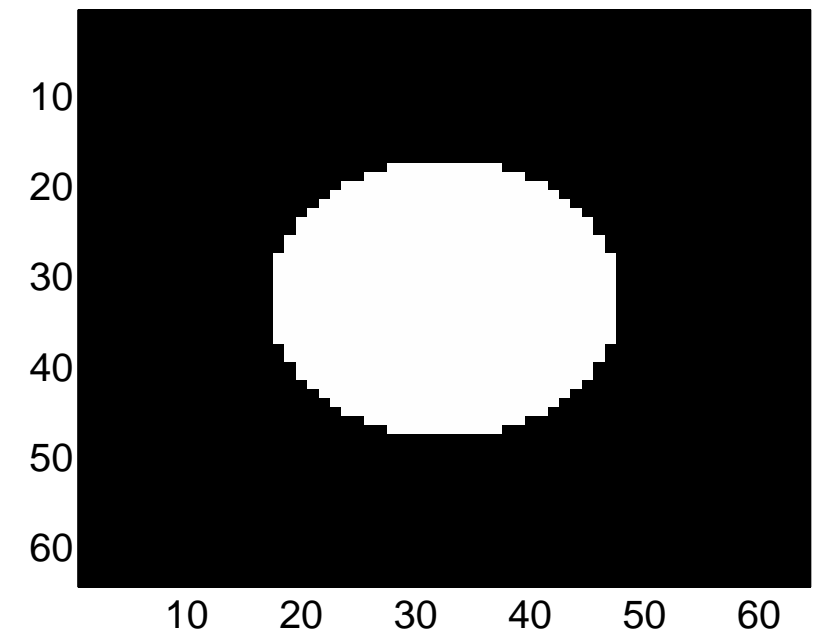

(a) Associated $\mu$ for the Levelset Function Below

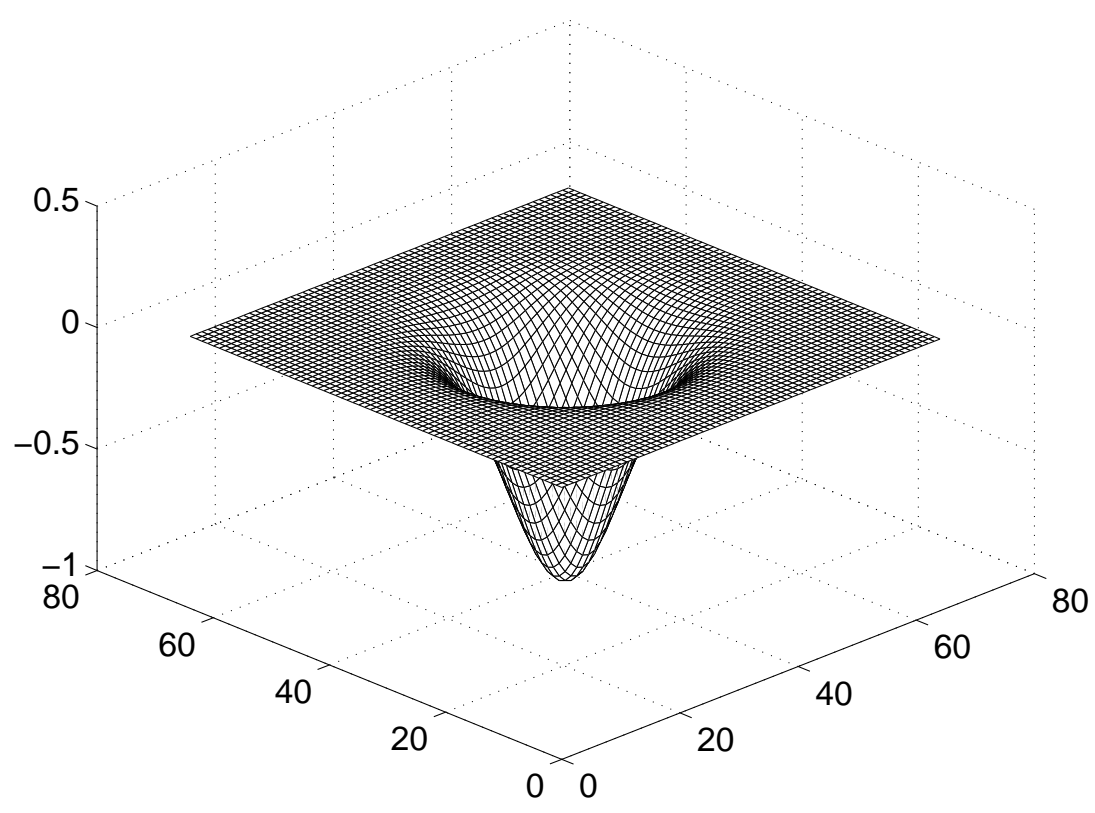

(b) The Initial Levelset Function

Fig. 8. Initial Levelset Function 


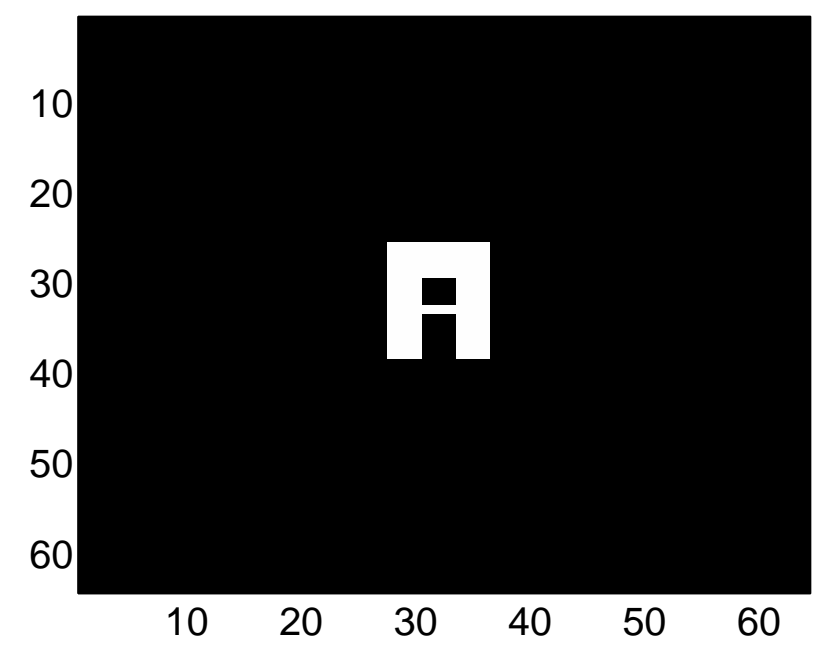

(a) Associated $\mu$ for the Levelset Function Below

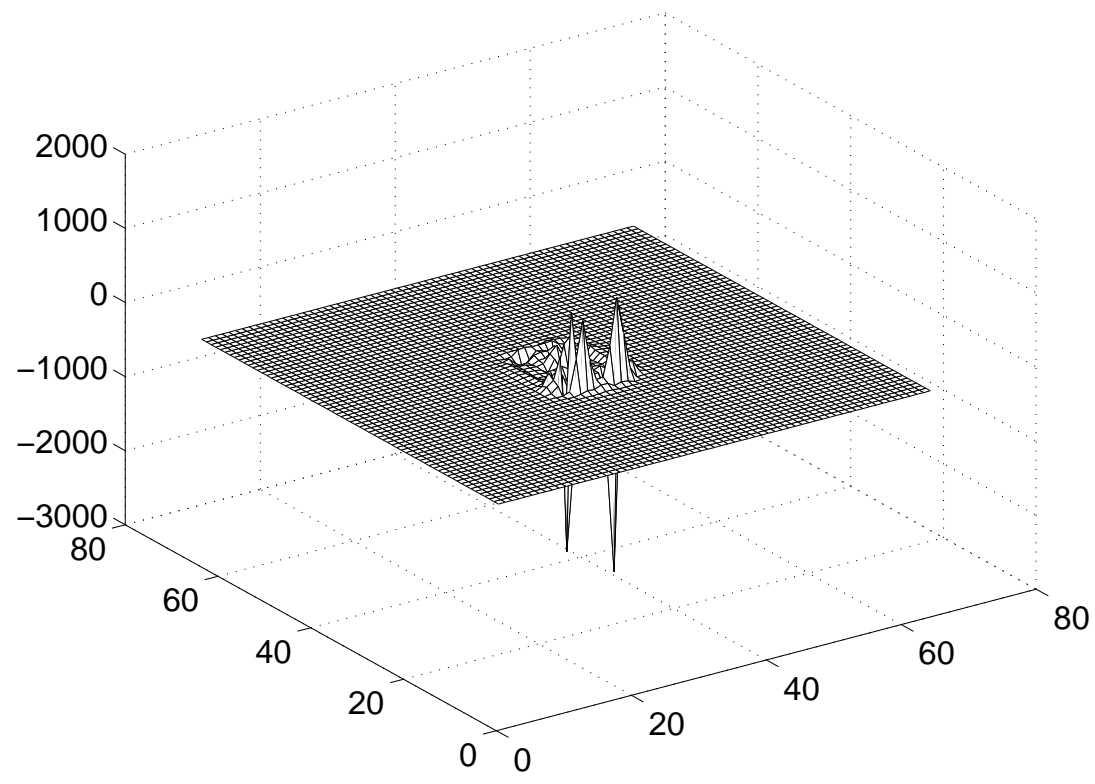

(b) The Terminal Levelset Function for the A-shape Reconstruction

Fig. 9. Final Levelset Function 
Although the result is good, it is not stable in the sense that if a small amount of noise is added to the data $\varphi$, the reconstruction deteriorates. To see the instability of this problem, we add blurred noise in Figure 10 to the energy fluence rate $\varphi$. Figure 11 shows reconstructions of the simple block image, with varying amount of noise. We observe that the reconstruction deteriorates rapidly when we add a small amount of noise.

We will address this problem with regularization by a total variation penalty in the next section.

\section{Levelset Method with Total Variation Regularization}

As we did in the gradient descent method, one way to reduce noise sensitivity in the reconstructions is to add a total variation regularization. That is, we consider the new minimization problem becomes

$$
\min _{\mu \in X}\|F(\mu)-\varphi\|_{L^{2}(\Omega)}^{2}+\beta \int_{\Omega} h_{\epsilon}(|\nabla \mu|)
$$

The regularization parameter $\beta$ can be varied to get the best reconstruction. Figure 12 shows the reconstruction of the simple block image with the regularization parameter $\beta=0.01$ and the smoothing parameter $\epsilon=0.001$, with same real solution as in Figure 4(a). Compared with Figure 11, Figure 12 shows much more stable reconstructions. Even though the results are good for the simple block shape, which has relatively small total variation, the reconstruction of images for which the total variation is large are not necessarily good. As illustrated in Figure 13, the reconstructions with and without total variation are not much different. Figure 13(c) was the "best" reconstruction obtained for several values of $\beta$. 


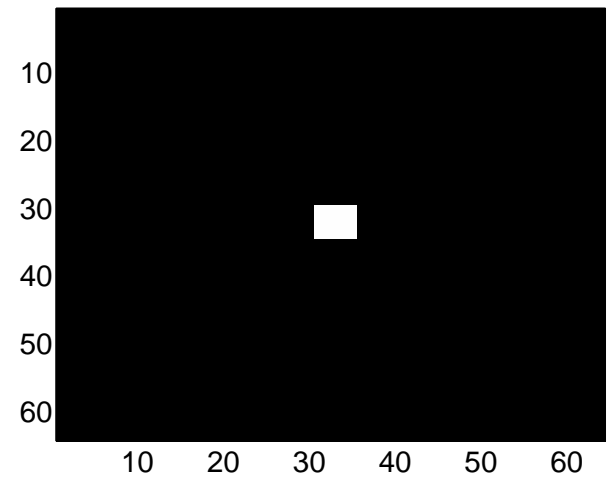

(a) Real Solution

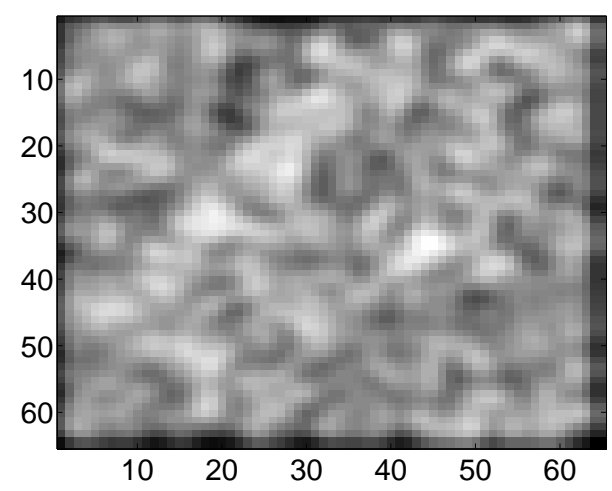

(b) Blurred Noise

Fig. 10. Blocky Solution and Blurred Noise 


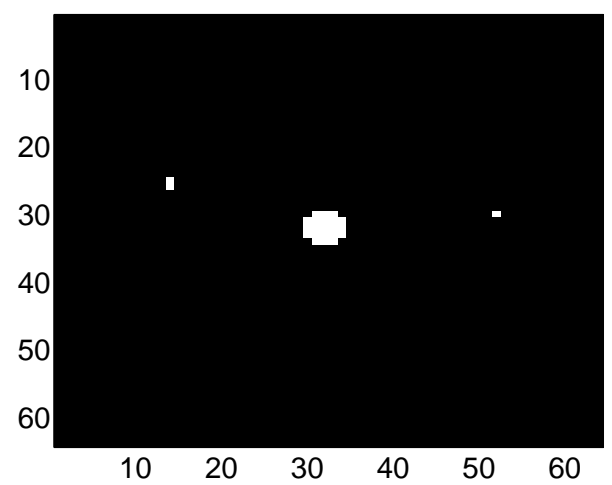

(a) with $2 \%$ noise

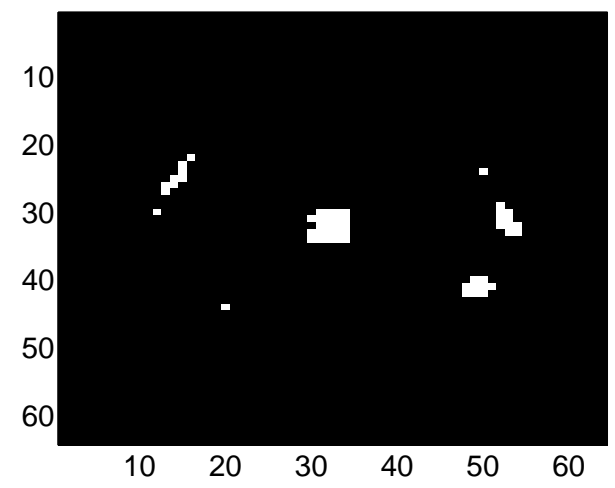

(b) with $5 \%$ noise

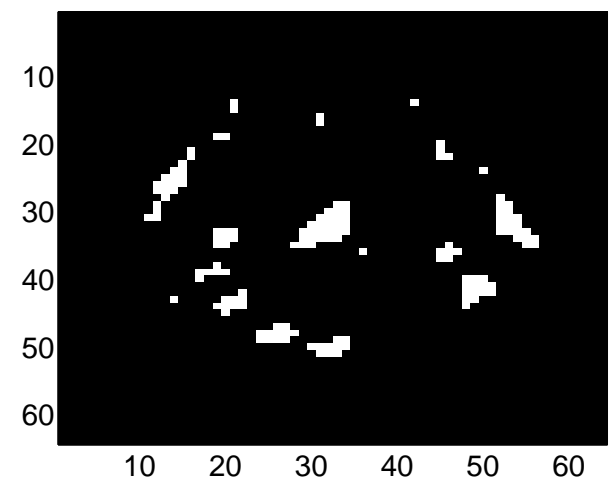

(c) with $10 \%$ noise

Fig. 11. Levelset Reconstructions with Noisy Data 


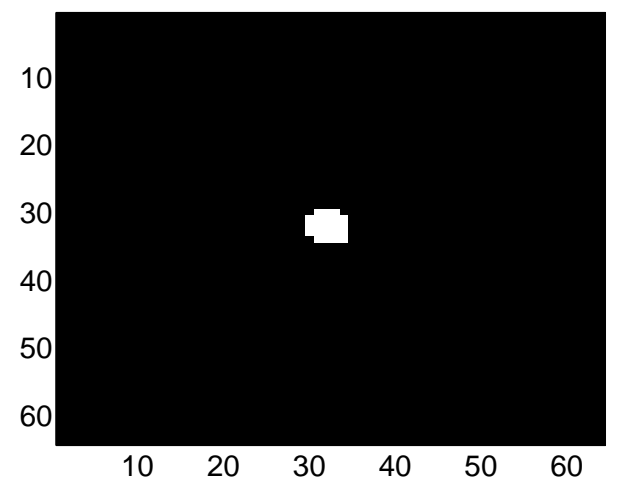

(a) $2 \%$ noise

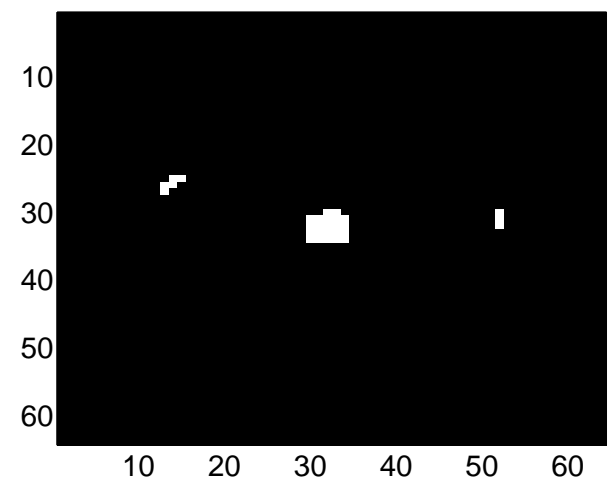

(b) $5 \%$ noise

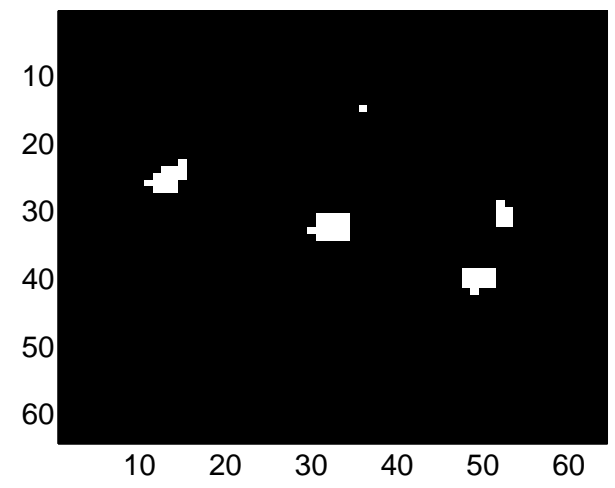

(c) $10 \%$ noise

Fig. 12. Simple Block Reconstructions with Total Variation Regularization 


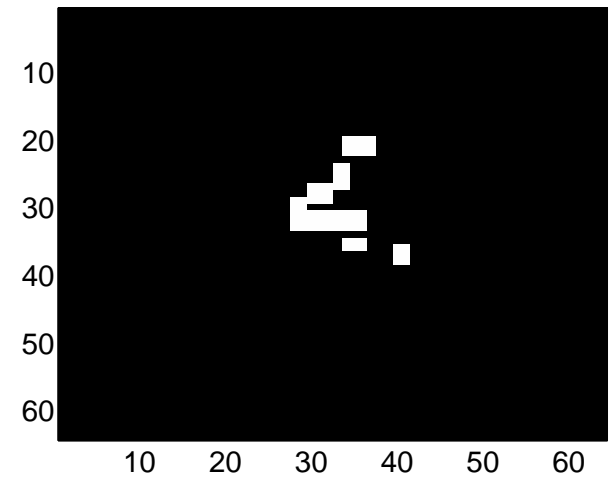

(a) real solution

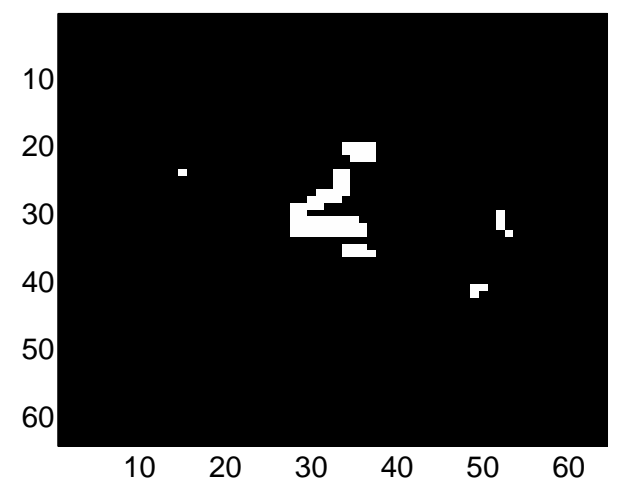

(b) $2 \%$ noise without total variation

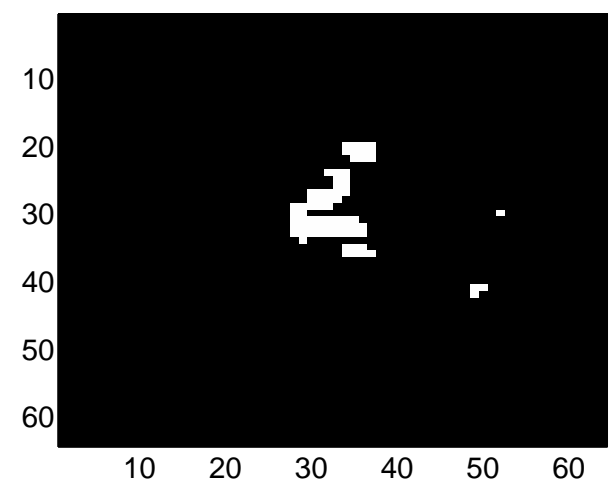

(c) $2 \%$ noise with total variation

Fig. 13. Complicated Shape Reconstruction with Total VariationRegularization 


\section{CHAPTER V}

\section{SUMMARY AND CONCLUSIONS}

In this thesis, we have studied the problem of ultrasound-modulated optical tomography, mostly from a mathematical point of view. Our aim has been to understand the basic principles of the reconstruction problem, using simplified mathematical models where necessary. To this end, we modeled photon transport in the biological medium by the diffusion approximation and the interaction of photons with the ultrasound column by a simple integral operator. Such an approach allows us to naturally separate the study of the reconstruction problem into two steps.

The first step is to solve a linear integral equation, and the second is to solve an inverse problem involving the diffusion equation. The linear integral equation has an unbounded inverse, but approximation methods involving regularization are well-known and very well understood.

The second step is formulated as a least-squares minimization problem. We have shown some analytic properties of the cost functional of the minimization problem, and proved that it admits solutions which are locally unique. We have also investigated several reconstruction methods and presented some numerical results for the inverse problem. We have used gradient descent and levelset methods. We have observed that the levelset method is quite fast and accurate. For stability, we have used total variation for regularizing both methods. The two methods each have advantages and disadvantages. The gradient descent approach allows the reconstruction of arbitrary absorption coefficient functions, but tends to give blurred, miscalibrated results. The levelset method gives faster, more accurate reconstructions, but assumes a two-component medium, and also has the potential to indicate false results (e.g., diseased tissue where none exists and vise versa) in the presence of noise. 
Overall improvements in UMOT will probably be driven more from advances in experimental techniques than from improved computational techniques. Nevertheless, we feel that there are significant opportunities to improve the reconstruction methods described here.

For future work, we could combine the two reconstruction steps as follows. Let $g_{0}$ be given measured data satisfying

$$
T u=g_{0},
$$

where $T$ is the linear integral operator (3.1) and $u=P \varphi$. Define

$$
H(\mu)=T(p(F(\mu)))
$$

where $p(u)(x)=P(x) u(x)$ is a multiplication operator. The function $H$ directly maps $\mu$ to the measured data $g_{0}$. Then a new minimization problem becomes

$$
\min _{\mu \in X} J_{0}(\mu)=\left\|H(\mu)-g_{0}\right\|_{L^{2}(\Omega)}^{2} .
$$

Since the new function $H$ is a composition of linear operators and $F, H$ satisfies the same analytic results which $F$ satisfies, e.g., existence and uniqueness of minimization problem, and the differentiability of $H$. Since the Fréchet derivative of $H$ is

$$
D H(\mu)(s)=T \circ p \circ(D F(\mu))(s),
$$

the Fréchet derivative of $J_{0}$ becomes

$$
D J_{0}(\mu)(s)=2 D F(\mu)^{*}\left(p \circ T^{*}\left(H(\mu)-g_{0}\right)\right) .
$$

This formulation has the advantage that only one problem needs to be solved, and only one regularization performed. More importantly, it implicitly "forces" the solution $\varphi$ of the first problem to be a solution of the diffusion equation, thereby eliminating 
problems with localized noise leading to spurious "diseased spots" in the reconstructions. The methods presented here will apply to minimization problem (5.1) with simple modifications.

The numerical experiments in this paper were done for the 2-dimensional case. But the main idea of the reconstruction method presented in this paper are not restricted to a 2-dimensional case. Thus, we think that it is possible to extend the method to the 3-dimensional case which is more realistic. For both 2-d and 3-d experiments, the data set is essentially two-dimensional. Thus, the primary new difficulty in the 3-d case is that some form of tomography or other experimental technique must be employed to get depth information.

Finally, to validate the ideas developed here, we want to carry out reconstructions with real experimental data. 


\section{REFERENCES}

[1] Rajendra Akerkar, Nonlinear Functional Analysis, Narosa Publishing House, New Delhi, 1999.

[2] A. P. Calderón, On an Inverse Boundary Value Problem, Seminar on Numerical Analysis and its Applications to Continuum Physics, Soc. Brasileria de Matematica, Rio de Janeiro, 1980.

[3] J. E. Dennis, R. Schnabel, Numerical Methods for Unconstrained Optimization and Nonlinear Equations, Prentice-Hall, Englewood Cliffs, NJ, 1983.

[4] David C. Dobson, Recovery of blocky images in electrical impedance tomography, in Inverse Problems in Medical Imaging and Nondestructive Testing, H.W. Engl, A.K. Louis, and W. Rundell, eds., Springer-Verlag, Wien, 1997.

[5] David C. Dobson and Fadil Santosa, Recovery of blocky images from noisy and blurred data, SIAM J. Appl. Math, 56 (1996), pp. 1181-1198.

[6] David C. Dobson and Fadil Santosa, An image-enhancement technique for electrical impedance tomography, Inverse Problems, 10 (1994), pp. 317-334.

[7] Oliver Dorn, A transport-backtransport method for optical tomography, Inverse Problems, 14 (1998), pp. 1107-1130.

[8] Oliver DoRn, Scattering and absorption transport sensitivity functions for optical tomography, Optics Express, 7 (2000), pp. 492-506.

[9] Oliver Dorn, Shape reconstruction in scattering media with voids using a transport model and level sets, Canadian Applied Mathematics Quarterly, to appear. 
[10] H. W. Engl, M. Hanke, A. Neubauer, Regularization of Inverse Problems, Kluwer Academic Publishers, Boston, 2000.

[11] D. Gilbarg N. S. Trudinger, Elliptic Partial Differential Equations of Second Order, Springer-Verlag, Berlin, Heidelberg, 1998.

[12] A. H. Hielscher and R. E. Alcouffe, Non-diffusive photon migration in homogeneous and heterogeneous tissues, SPIE, 2925 (1996), pp. 22-30.

[13] K. Ito, K. Kunisch AND Z. LI, Level-set function approach to an inverse interface problem, Inverse Problems, 17 (2001), pp. 1225-1242.

[14] M. Kempe, M. Larionov, D. Zaslavsky, A. Z. Genack, Acousto-optic tomography with multiply scattered light, J. Opt. Soc. Am. A., 14 (1997), pp. 11511158.

[15] IAN KNOWLES, Uniqueness for an elliptic inverse problem, SIAM J. Appl. Math., 59 (1999), pp. 1356-1370.

[16] Erwin Kreyszig, Introductory Functional Analysis with Applications, John Wiley \& Sons Inc., New York, 1978.

[17] W. Leutz, G. Maret, Ultrasonic modulation of multiply scattered light, Physic B, 204 (1995), pp. 14-19.

[18] A. Litman, D. Lesselier And F. Santosa, Reconstruction of a twodimensional binary obstacle by controlled evolution of a level-set, Inverse Problems, 14 (1998), pp. 685-706.

[19] R. Model, R. Hunlich, D. Richter, H. Rinneberg, H.Wabnitz, M. WALZEL, Imaging in random media: simulating light transport by numerical integration of the diffusion equation, SPIE, 2326, pp. 11-22. 
[20] S. Osher And J. Sethian, Fronts propagation with curvature dependent speed: Algorithm based on Hamilton-Jacobi formulations, Journal of Computational Physics, 56 (1998), pp. 12-49.

[21] Gerard R. Richter, An inverse problem for the steady state diffusion equation, SIAM J. Appl. Math., 41 (1981), pp. 210-221.

[22] Michael Renardy, Robert C. Rogers, An Introduction to Partial Differential Equations, Springer-Verlag, Berlin, Heidelberg, 1993.

[23] L. I. Rudin, S. Osher, And E. FAtami, Nonlinear total variation based noise removal algorithms, Physica D., 60 (1992), pp 259-268.

[24] FAdil Santosa, A level-set approach for inverse problems involving obstacles, ESIAM COCV, 1 (1996), pp. 17-33.

[25] L. Wang, S. L. Jacques And L. Zheng, MCML-Monte Carlo modeling of light transport in multi-layered tissues, Computer Methods and Programs in Biomedicine, 47 (1995), pp. 131-146.

[26] Lihong Wang, BMEN-620, Class notes, Department of Biomedical Engineering, Texas A\&M University, (2002).

[27] Gang Yao, Lihong V. Wang, Full-field mapping of ultrasonic field by lightsource-synchronized projection, J. Accoust. Soc. Am, 106 (1999), pp. 36-40.

[28] Gang Yao, Lihong V. WAng, Theoretical and experimental studies of ultrasound-modulated optical tomography in biological tissue, Applied Optics, vol 39 (2000), pp. 659-664.

[29] E. ZeIDler, Applied Functional Analysis, Springer, New York, 2nd edition, 1997. 


\section{VITA}

The author of this dissertation, Haewon Nam, received her B.S. degree in mathematics from Ewha Women's University in 1996 and M.S. degree in mathematics in 1998. Throughout her career she has worked in operator theory. Her research interests include inverse problem solving, optical tomography, and image processing. The author may be reached at Daerim Acrovil B-2503, Kangnamku Dogukdong, Seoul, Korea.

The typist for this dissertation was Haewon Nam. 